TRANSACTIONS OF THE

AMERICAN MATHEMATICAL SOCIETY

Volume 365, Number 12, December 2013, Pages 6697-6729

S 0002-9947(2013)05960-0

Article electronically published on July 26, 2013

\title{
ALGEBRAIC TRANSFORMATIONS OF HYPERGEOMETRIC FUNCTIONS AND AUTOMORPHIC FORMS ON SHIMURA CURVES
}

\author{
FANG-TING TU AND YIFAN YANG
}

\begin{abstract}
In this paper, we will obtain new algebraic transformations of the ${ }_{2} F_{1}$-hypergeometric functions. The main novelty in our approach is the interpretation of identities among ${ }_{2} F_{1}$-hypergeometric functions as identities among automorphic forms on different Shimura curves.
\end{abstract}

\section{INTRODUCTION}

For a real number $a$ and a nonnegative integer $n$, let

$$
(a)_{n}= \begin{cases}1, & \text { if } n=0, \\ a(a+1) \ldots(a+n-1), & \text { if } n \geq 1\end{cases}
$$

be the Pochhammer symbol. Recall that, for real numbers $a, b, c$ with $c \neq 0,-1,-2$, $\ldots$, the ${ }_{2} F_{1}$-hypergeometric function is defined by

$$
{ }_{2} F_{1}(a, b ; c ; z)=\sum_{n=0}^{\infty} \frac{(a)_{n}(b)_{n}}{(c)_{n} n !} z^{n}
$$

for $z \in \mathbb{C}$ with $|z|<1$. The hypergeometric function is a solution of the differential equation

$$
\theta(\theta+c-1) F-z(\theta+a)(\theta+b) F=0, \quad \theta=z \frac{d}{d z} .
$$

Hypergeometric functions arise naturally in many branches of mathematics. For example, the periods

$$
\int_{1}^{\infty} \frac{d x}{\sqrt{x(x-1)(x-\lambda)}}
$$

of the Legendre family of elliptic curves $E_{\lambda}: y^{2}=x(x-1)(x-\lambda)$ can be expressed as

Also, it is well known that

$$
{ }_{2} F_{1}\left(\frac{1}{2}, \frac{1}{2} ; 1 ; \lambda\right) \text {. }
$$

$$
E_{4}(\tau)={ }_{2} F_{1}\left(\frac{1}{12}, \frac{5}{12} ; 1 ; \frac{1728}{j(\tau)}\right)^{4},
$$

where $E_{4}(\tau)$ is the Eisenstein series of weight 4 on $\operatorname{SL}(2, \mathbb{Z})$ and $j(\tau)$ is the elliptic $j$-function.

Received by the editors December 12, 2011 and, in revised form, September 10, 2012.

2010 Mathematics Subject Classification. Primary 11F12; Secondary 11G18, 33C05.

The authors were partially supported by Grant 99-2115-M-009-011-MY3 of the National Science Council, Taiwan (R.O.C.).

(C) 2013 American Mathematical Society Reverts to public domain 28 years from publication 
In this paper, we are concerned with algebraic transformations of hypergeometric functions, that is, identities of the form

$$
{ }_{2} F_{1}(a, b ; c ; z)=R(z){ }_{2} F_{1}\left(a^{\prime}, b^{\prime} ; c^{\prime} ; S(z)\right)
$$

with suitable parameters $a, b, c, a^{\prime}, b^{\prime}, c^{\prime}$ and algebraic functions $R(z)$ and $S(z)$. If $w=S(z)$ is of degree $m$ over the field $\mathbb{C}(z)$ or if $z$ is of degree $m$ over the field $\mathbb{C}(w)$, we say the algebraic transformation has degree $m$. Two examples of algebraic transformations of degree 1 are given by

${ }_{2} F_{1}(a, b ; c ; z)=(1-z)^{c-a-b}{ }_{2} F_{1}(c-a, c-b ; c ; z)=(1-z)^{-a}{ }_{2} F_{1}\left(a, c-b ; c ; \frac{z}{z-1}\right)$.

These identities can be easily proved using the well-known result in the classical analysis that a Fuchsian differential equation with precisely three singularities at 0,1 , and $\infty$ is completely determined by the local exponents at these three points.

Beyond transformations of degree 1, one of the simplest examples is Kummer's quadratic transformation

$$
{ }_{2} F_{1}\left(2 a, 2 b ; a+b+\frac{1}{2} ; z\right)={ }_{2} F_{1}\left(a, b ; a+b+\frac{1}{2} ; 4 z(1-z)\right),
$$

valid for any real numbers $a, b$ with $a+b+1 / 2 \neq 0,-1,-2, \ldots$ In [3], Goursat gave more than 100 algebraic transformations of degrees $2,3,4,6$. One such example is

$$
{ }_{2} F_{1}\left(a, a+\frac{1}{3} ; \frac{1}{2} ; \frac{z(9-8 z)^{2}}{(4 z-3)^{3}}\right)=\left(1+\frac{z}{3}\right)^{3 a}{ }_{2} F_{1}\left(3 a, a+\frac{1}{6} ; \frac{1}{2} ; z\right)
$$

of degree 3. (See entry (96) on page 132 of [3].) More recently, Vidūnas [10] gave dozens of new algebraic transformations of degrees $6,8,9,10,12$. For example, he showed that if we set $\beta= \pm \sqrt{-2}$,

$$
S(z)=\frac{4 z(z-1)(8 \beta z+7-4 \beta)^{8}}{\left(2048 \beta z^{3}-3072 \beta z^{2}-3264 z^{2}+912 \beta z+3264 z+56 \beta-17\right)^{3}},
$$

and

$$
R(z)=\left(1+\frac{16}{9}(4-17 \beta) z-\frac{64}{243}(167-136 \beta) z^{2}+\frac{2048}{6561}(112-17 \beta) z^{3}\right)^{-1 / 16},
$$

then

$$
{ }_{2} F_{1}\left(\frac{5}{24}, \frac{13}{24} ; \frac{7}{8} ; z\right)=R(z){ }_{2} F_{1}\left(\frac{1}{48}, \frac{17}{48} ; \frac{7}{8} ; S(z)\right),
$$

which is a transformation of degree 10. (See (32) of [10].) Vidūnas' examples usually involve Gröbner-basis computation. This is perhaps one of the reasons why Goursat could not find these transformations.

In this paper, we will present several new algebraic transformations. For example, one of our favorite identities is

$$
\begin{aligned}
{ }_{2} F_{1}\left(\frac{1}{20},\right. & \left.\frac{1}{4} ; \frac{4}{5} ; \frac{64 z\left(1-z-z^{2}\right)^{5}}{\left(1-z^{2}\right)\left(1+4 z-z^{2}\right)^{5}}\right) \\
& =\left(1-z^{2}\right)^{1 / 20}\left(1+4 z-z^{2}\right)^{1 / 4}{ }_{2} F_{1}\left(\frac{3}{10}, \frac{2}{5} ; \frac{9}{10} ; z^{2}\right) .
\end{aligned}
$$

The main novelty in our approach is the interpretation of ${ }_{2} F_{1}$-hypergeometric functions as automorphic forms on Shimura curves. Then proving identities such as the one above amounts to showing that two certain automorphic forms on two Shimura 
curves are equal. This point of view is especially useful in determining the function $R(z)$ in (1). We will review the basic definitions regarding Shimura curves and their automorphic forms in the next section.

\section{Preliminaries}

The materials in this section are mostly taken from [11].

Quaternion algebras. A quaternion algebra $B$ over a field $K$ is a central simple algebra of dimension 4 over $K$. (Here central means that the center of the algebra is $K$ and simple means that $B$ has no proper nontrivial two-sided ideals.) If the characteristic of $K$ is not 2, then one can show that there are elements $i$ and $j$ in $B$ and $a, b \in K^{\times}$such that

$$
i^{2}=a, \quad j^{2}=b, \quad i j=-j i,
$$

and $B=K+K i+K j+K i j$. In this case, we denote this algebra by $\left(\frac{a, b}{K}\right)$. For example, we have $M(2, K) \simeq\left(\frac{1,1}{K}\right)$ and $\left(\frac{-1,-1}{\mathbb{R}}\right)$ is the set of Hamilton's quaternions. Moreover, for $\alpha=a_{0}+a_{1} i+a_{2} j+a_{3} i j \in B$, we set $\bar{\alpha}=a_{0}-a_{1} i-a_{2} j-a_{3} i j$. Then the reduced trace $\operatorname{tr}(\alpha)$ is defined to be $\alpha+\bar{\alpha}=2 a_{0} \in K$ and the reduced norm $n(\alpha)$ is defined to be $\alpha \bar{\alpha}=a_{0}^{2}-a_{1}^{2} a-a_{2}^{2} b+a_{3}^{2} a b \in K$.

If $K=\mathbb{C}$, then up to isomorphisms, there is only one quaternion algebra over $\mathbb{C}$, which is $M(2, \mathbb{C})$. If $K=\mathbb{R}$ or a non-Archimedean local field, then up to isomorphism, there are only two quaternion algebras. One is $M(2, K)$ and the other is a division algebra.

Now assume that $K$ is a number field. Let $v$ be a place of $K$ and $K_{v}$ be the completion of $K$ with respect to $v$. If the localization $B \otimes_{K} K_{v}$ is isomorphic to $M\left(2, K_{v}\right)$, we say $B$ splits at $v$. If $B \otimes_{K} K_{v}$ is isomorphic to a division algebra, we say $B$ ramifies at $v$. It is known that the number of ramified places is finite and in fact an even integer. The product of finite ramified places is called the discriminant of the quaternion algebra.

Still assume that $K$ is a number field. Let $R$ be its ring of integers. An order in $B$ is a finite generated $R$-module that is also a ring with unity containing a basis for $B$. An order is maximal if it is not properly contained in another order. An Eichler order is the intersection of two maximal orders. For example, if $B=M(2, \mathbb{Q})$, then $M(2, \mathbb{Z})$ is a maximal order and $\left(\begin{array}{c}\mathbb{Z} \\ N \mathbb{Z}\end{array}\right)=M(2, \mathbb{Z}) \cap\left(\begin{array}{ll}1 & 0 \\ 0 & N\end{array}\right) M(2, \mathbb{Z})\left(\begin{array}{ll}1 & 0 \\ 0 & N\end{array}\right)^{-1}$ is an Eichler order.

Shimura curves. To define a Shimura curve, we assume that $K$ is a totally real number field and take a quaternion algebra $B$ over $K$ that splits at exactly one infinite place, that is,

$$
B \otimes_{\mathbb{Q}} \mathbb{R} \simeq M(2, \mathbb{R}) \times \mathbb{H}^{[K: \mathbb{Q}]-1},
$$

where $\mathbb{H}$ is Hamilton's quaternion algebra $\left(\frac{-1,-1}{\mathbb{R}}\right)$. Then, up to conjugation, there is a unique embedding $\iota$ of $B$ into $M(2, \mathbb{R})$. Note that we have $n(\alpha)=\operatorname{det} \iota(\alpha)$ for all $\alpha \in B$.

Let $\mathcal{O}$ be an order and $\mathcal{O}_{1}^{*}=\{\alpha \in \mathcal{O}: n(\alpha)=1\}$ be the norm-one group of $\mathcal{O}$. Then the image $\Gamma(\mathcal{O})$ of $\mathcal{O}_{1}^{*}$ under the embedding $\iota$ is a discrete subgroup of $\operatorname{SL}(2, \mathbb{R})$. Let $\Gamma(\mathcal{O})$ act on the upper half-plane $\mathfrak{H}$ in the usual manner,

$$
\left(\begin{array}{ll}
a & b \\
c & d
\end{array}\right): \tau \longmapsto \frac{a \tau+b}{c \tau+d}
$$


Then the quotient space $\Gamma(\mathcal{O}) \backslash \mathfrak{H}$ is called the Shimura curve associated to $\mathcal{O}$. For example, if $B=M(2, \mathbb{Q})$ and $\mathcal{O}=M(2, \mathbb{Z})$, then $\Gamma(\mathcal{O})=\operatorname{SL}(2, \mathbb{Z})$ and $\Gamma(\mathcal{O}) \backslash \mathfrak{H}$ is just the usual modular curve $Y_{0}(1)$. Thus, Shimura curves are generalizations of classical modular curves and they are moduli spaces of certain abelian surfaces with quaternionic multiplication [5]. In a broader setting, if $\Gamma$ is any discrete subgroup of $\operatorname{SL}(2, \mathbb{R})$ commensurable with $\Gamma(\mathcal{O})$, then the quotient space $\Gamma \backslash \mathfrak{H}$ will also be called a Shimura curve.

An element $\gamma$ of $\Gamma(\mathcal{O})$ is parabolic, elliptic, or hyperbolic, according to whether $|\operatorname{tr}(\gamma)|=2,|\operatorname{tr}(\gamma)|<2$, or $|\operatorname{tr}(\gamma)|>2$. The fixed point of a parabolic element is called a cusp. This can appear only when $B=M(2, \mathbb{Q})$. The fixed point $\tau$ of an elliptic element in $\mathfrak{H}$ is called an elliptic point of order $n$, where $n$ is the order of the isotropy subgroup of $\tau$ in $\Gamma(\mathcal{O}) / \pm 1$.

Note that if $B \neq M(2, \mathbb{Q})$, then the quotient space $\Gamma(\mathcal{O}) \backslash \mathfrak{H}$ is a compact Riemann surface, which we denote by $X(\mathcal{O})$. If $B=M(2, \mathbb{Q})$, we compactify the Riemann surface $\Gamma(\mathcal{O}) \backslash \mathfrak{H}$ by adding cusps, and the resulting compact surface will also be denoted by $X(\mathcal{O})$.

Now suppose that the compact Riemann surface $X(\mathcal{O})$ has genus $g$. Then a classical result says that there exist hyperbolic elements $A_{1}, \ldots, A_{g}, B_{1}, \ldots, B_{g}$, and elliptic or parabolic elements $C_{1}, \ldots, C_{r}$ that generate $\Gamma(\mathcal{O}) / \pm 1$ with the single relation

$$
\left[A_{1}, B_{1}\right] \ldots\left[A_{g}, B_{g}\right] C_{1} \ldots C_{r}=\mathrm{Id}
$$

where $\left[A_{i}, B_{i}\right]=A_{i} B_{i} A_{i}^{-1} B_{i}^{-1}$ is the commutator of $A_{i}$ and $B_{i}$. (See 4, Chapter 4].) We let $\left(g ; e_{1}, \ldots, e_{r}\right)$ be the signature of $X(\mathcal{O})$.

Triangle groups. Suppose that a Shimura curve $X(\mathcal{O})$ has signature $\left(0 ; e_{1}, e_{2}, e_{3}\right)$. Then we say $\Gamma(\mathcal{O})$ is an arithmetic triangle group. The complete lists of all arithmetic triangle groups and their commensurability classes were determined by Takeuchi [7, 8].

If we cut each fundamental domain of an arithmetic triangle group $\Gamma(\mathcal{O})$ into 2 halves in a suitable way, then the fundamental half-domains give a tessellation of the upper half-plane $\mathfrak{H}$ by congruent triangles with internal angles $\pi / e_{1}, \pi / e_{2}$, and $\pi / e_{3}$. The following figure shows the tessellation of the unit disc, which is conformally equivalent to $\mathfrak{H}$ through $\tau \rightarrow(\tau-i) /(\tau+i)$, by fundamental halfdomains of the arithmetic triangle group $(0 ; 2,3,7)$.

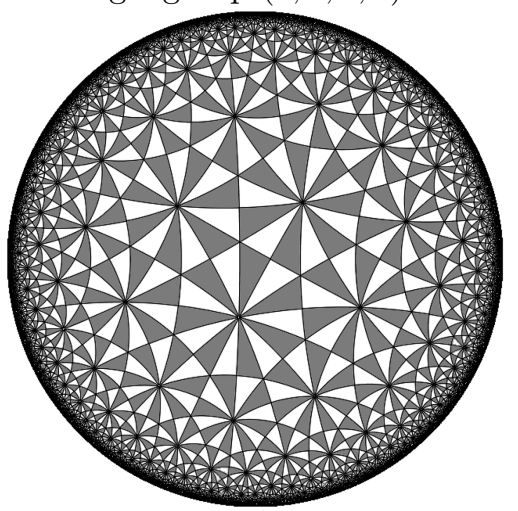

Here each triangle represents a fundamental half-domain. Any combination of a grey triangle with a neighboring white triangle will be a fundamental domain for the 
triangle group $(0 ; 2,3,7)$. The triangle group $(0 ; 2,3,7)$ and its associated Shimura curve have been studied in detail in [2].

In general, for any discrete subgroup $\Gamma$ of $\operatorname{SL}(\mathbb{R})$ such that $\Gamma \backslash \mathfrak{H}$ has finite volume, we can define its signature in the same way. If the signature is $\left(0 ; e_{1}, e_{2}, e_{3}\right)$, then we say $\Gamma$ is a (hyperbolic) triangle group. (There are also notions of parabolic and elliptic triangle groups, corresponding to tessellation of $\mathbb{C}$ and $\mathbb{P}^{1}(\mathbb{C})$, respectively.)

Automorphic forms on Shimura curves. The definition of an automorphic form on Shimura curves is the same as that of a modular form on classical modular curves.

For simplicity, we assume that $B \neq M(2, \mathbb{Q})$ so that we do not need to consider cusps. Then an automorphic form of weight $k$ on $\Gamma(\mathcal{O})$ is a holomorphic function $f: \mathfrak{H} \rightarrow \mathbb{C}$ such that

$$
f\left(\frac{a \tau+b}{c \tau+d}\right)=(c \tau+d)^{k} f(\tau)
$$

for all $\left(\begin{array}{ll}a & b \\ c & d\end{array}\right) \in \Gamma(\mathcal{O})$ and all $\tau \in \mathfrak{H}$. The space of automorphic forms of weight $k$ on $\Gamma(\mathcal{O})$ will be denoted by $S_{k}(\mathcal{O})$. Also, if a meromorphic function $f: \mathfrak{H} \rightarrow \mathbb{C}$ satisfies (44) with $k=0$, we say $f$ is an automorphic function. If $X(\mathcal{O})$ has genus 0 , we call an automorphic function a Hauptmodul if it generates the field of automorphic functions on $\Gamma(\mathcal{O})$.

Using the Riemann-Roch formula, one can calculate the dimension of $S_{k}(\mathcal{O})$.

Proposition 1 ([6, Theorem 2.23]). Assume that $B \neq M(2, \mathbb{Q})$. Suppose that the Shimura curve $X(\mathcal{O})$ associated to an order $\mathcal{O}$ in $B$ has signature $\left(g ; e_{1}, \ldots, e_{r}\right)$. Then for even integers $k$, we have

$$
\operatorname{dim} S_{k}(\mathcal{O})= \begin{cases}0, & \text { if } k<0, \\ 1, & \text { if } k=0, \\ g, & \text { if } k=2, \\ (k-1)(g-1)+\sum_{j=1}^{r}\left\lfloor\frac{k}{2}\left(1-\frac{1}{e_{j}}\right)\right\rfloor, & \text { if } k \geq 4 .\end{cases}
$$

The dimension formula for the case $B=M(2, \mathbb{Q})$ is slightly different.

In the case $B=M(2, \mathbb{Q})$, there are many methods to construct modular forms, such as the Eisenstein series, theta series, Dedekind eta function, etc. In practice, most explicit methods for modular curves rely on the Fourier expansions of modular forms and modular functions, i.e., the expansions with respect to the local parameter at the cusp $\infty$. However, in the case $B \neq M(2, \mathbb{Q})$, because of the lack of cusps on Shimura curves, very few explicit methods are available for Shimura curves. Some of the few methods are given in [1] by Bayer and Travesa and [12] by the second author of the present paper.

One of the key ideas in 12 is the following characterization of $S_{k}(\mathcal{O})$. Here we assume that the quaternion algebra is not $M(2, \mathbb{Q})$.

Proposition 2 ([12, Theorem 4, Propositions 1 and 6]). Assume that a Shimura curve $X$ has genus zero with elliptic points $\tau_{1}, \ldots, \tau_{r}$ of order $e_{1}, \ldots, e_{r}$, respectively. Let $t(\tau)$ be a Hauptmodul of $X$ and set $a_{i}=t\left(\tau_{i}\right), i=1, \ldots, r$. For a positive even 
integer $k \geq 4$, let

$$
d_{k}=\operatorname{dim} S_{k}(\mathcal{O})=1-k+\sum_{j=1}^{r}\left\lfloor\frac{k}{2}\left(1-\frac{1}{e_{j}}\right)\right\rfloor .
$$

Then a basis for the space of automorphic forms of weight $k$ on $X$ is

$$
t^{\prime}(\tau)^{k / 2} t(\tau)^{j} \prod_{i=1, a_{i} \neq \infty}^{r}\left(t(\tau)-a_{i}\right)^{-\left\lfloor k\left(1-1 / e_{i}\right) / 2\right\rfloor}, \quad j=0, \ldots, d_{k}-1 .
$$

Moreover, the functions $t^{\prime}(\tau)^{1 / 2}$ and $\tau t^{\prime}(\tau)^{1 / 2}$, as functions of $t$, satisfy the differential equation

$$
f^{\prime \prime}+Q(t) f=0
$$

where

$$
Q(t)=\frac{1}{4} \sum_{j=1, a_{j} \neq \infty}^{r} \frac{1-1 / e_{j}^{2}}{\left(t-a_{j}\right)^{2}}+\sum_{j=1, a_{j} \neq \infty}^{r} \frac{B_{j}}{t-a_{j}}
$$

for some constants $B_{j}$. Moreover, if $a_{j} \neq \infty$ for all $j$, then the constants $B_{j}$ satisfy

$$
\sum_{j=1}^{r} B_{j}=\sum_{j=1}^{r}\left(a_{j} B_{j}+\frac{1}{4}\left(1-1 / e_{j}^{2}\right)\right)=\sum_{j=1}^{r}\left(a_{j}^{2} B_{j}+\frac{1}{2} a_{j}\left(1-1 / e_{j}^{2}\right)\right)=0 .
$$

Also, if $a_{r}=\infty$, then $B_{j}$ satisfy

$$
\sum_{j=1}^{r-1} B_{j}=0, \quad \sum_{j=1}^{r-1}\left(a_{j} B_{j}+\frac{1}{4}\left(1-1 / e_{j}^{2}\right)\right)=\frac{1}{4}\left(1-1 / e_{r}^{2}\right) .
$$

Remark 3. In [12, the differential equation $f^{\prime \prime}+Q(t) f=0$ is called the Schwarzian differential equation associated to $t$ because $Q(t)$ is related to the Schwarzian derivative by the relation

$$
2 Q(t) t^{\prime}(\tau)^{2}+\{t, \tau\}=0
$$

where

$$
\{t, \tau\}=\frac{t^{\prime \prime \prime}(\tau)}{t^{\prime}(\tau)}-\frac{3}{2}\left(\frac{t^{\prime \prime}(\tau)}{t^{\prime}(\tau)}\right)^{2}
$$

is the Schwarzian derivative. In general, in literature [1, if $f$ is a thrice-differentiable function of $z$, then

$$
D(f, z):=-\frac{\{f, z\}}{2 f^{\prime}(z)^{2}}
$$

is called the automorphic derivative associated to $f$ and $z$. In the case $f$ is an automorphic function on a Shimura curve, then $D(f, \tau)$ is also an automorphic function. In particular, if $t$ is a Hauptmodul on a Shimura curve of genus 0 , then $Q(t)=D(t, \tau)$ is a rational function of $t$.

The upshot of this result is that it is often possible to determine the differential equation without explicitly constructing a Hauptmodul. For example, if $\Gamma(\mathcal{O})$ is a triangle group with signature $\left(0 ; e_{1}, e_{2}, e_{3}\right)$, then there always exists a (unique) Hauptmodul $t$ with $a_{1}=0, a_{2}=1$, and $a_{3}=\infty$. Then the relations between $B_{j}$ uniquely determine the differential equation. In general, one can usually use coverings between Shimura curves of genus 0 to determine the differential equation. This is done by Bayer and Travesa [1] and by the first author [9] of the present paper for many Shimura curves of genus 0 associated to orders in quaternion algebras over $\mathbb{Q}$. Once the differential equation is determined, one can express automorphic forms 
in terms of $t$-series and then study properties of automorphic forms using these $t$ series. For example, in 12 the second author devised a method to compute Hecke operators on these $t$-series.

In the case of triangle groups, since the number of singularities of the differential equation is 3 , the differential equation is essentially a hypergeometric differential equation.

Proposition 4 ([12, Theorem 9]). Assume that a Shimura curve $X$ has signature $\left(0 ; e_{1}, e_{2}, e_{3}\right)$. Let $t(\tau)$ be the Hauptmodul of $X$ with values 0,1 , and $\infty$ at the elliptic points of order $e_{1}, e_{2}$, and $e_{3}$, respectively. Let $k \geq 4$ be an even integer. Then a basis for the space of automorphic forms of weight $k$ on $X$ is given by

$$
t^{\left\{k\left(1-1 / e_{1}\right) / 2\right\}}(1-t)^{\left\{k\left(1-1 / e_{2}\right) / 2\right\}} t^{j}\left({ }_{2} F_{1}(a, b ; c ; t)+C t^{1 / e_{1}}{ }_{2} F_{1}\left(a^{\prime}, b^{\prime}, c^{\prime} ; t\right)\right)^{k},
$$

$j=0, \ldots,\left\lfloor k\left(1-1 / e_{1}\right) / 2\right\rfloor+\left\lfloor k\left(1-1 / e_{2}\right) / 2\right\rfloor+\left\lfloor k\left(1-1 / e_{3}\right) / 2\right\rfloor-k$, for some constant $C$, where for a rational number $x$, we let $\{x\}$ denote the fractional part of $x$,

$$
a=\frac{1}{2}\left(1-\frac{1}{e_{1}}-\frac{1}{e_{2}}-\frac{1}{e_{3}}\right), \quad b=a+\frac{1}{e_{3}}, \quad c=1-\frac{1}{e_{1}}
$$

and

$$
a^{\prime}=a+\frac{1}{e_{1}}, \quad b^{\prime}=b+\frac{1}{e_{1}}, \quad c^{\prime}=c+\frac{2}{e_{1}} .
$$

For general Shimura curves of genus 0, the following properties of automorphic derivatives are very useful in determining the Schwarzian differential equation associated to a Hauptmodul.

Proposition 5. Automorphic derivatives have the following properties.

(1) $D((a z+b) /(c z+d), z)=0$ for all $\left(\begin{array}{ll}a & b \\ c & d\end{array}\right) \in \mathrm{GL}(2, \mathbb{C})$.

(2) $D(g \circ f, z)=D(g, f(z))+D(f, z) /(d g / d f)^{2}$.

Proposition 6. Let $z(\tau)$ be a Hauptmodul for a Shimura curve $X(\mathcal{O})$ of genus 0 . Let $R(x) \in \mathbb{C}(x)$ be the rational function such that the automorphic derivative $Q(z)=D(z, \tau)$ is equal to $R(z)$. Assume that $\gamma$ is an element of $\operatorname{SL}(2, \mathbb{R})$ normalizing the norm-one group of $\mathcal{O}$ and let $\sigma$ be the automorphism of $X(\mathcal{O})$ induced by $\gamma$. If $\sigma: z \mapsto(a z+b) /(c z+d)$, then $R(x)$ satisfies

$$
\frac{(a d-b c)^{2}}{(c x+d)^{4}} R\left(\frac{a x+b}{c x+d}\right)=R(x) .
$$

Proof. We shall compute $D(z(\gamma \tau), \tau)$ in two ways. By Proposition 5, we have

$$
D(z(\gamma \tau), \tau)=D\left(\frac{a z(\tau)+b}{c z(\tau)+d}, z(\tau)\right)+\frac{D(z(\tau), \tau)}{(d z(\gamma \tau) / d z(\tau))^{2}}=0+\frac{(c z+d)^{4} R(z)}{(a d-b c)^{2}} .
$$

On the other hand, by the same proposition, we also have

$$
D(z(\gamma \tau), \tau)=D(z(\gamma \tau), \gamma \tau)+\frac{D(\gamma \tau, \tau)}{(d z(\gamma \tau) / d \gamma \tau)^{2}}=R(z(\gamma \tau))=R\left(\frac{a z+b}{c z+d}\right) .
$$

Comparing the two expressions, we get the formula. 
Algebraic transformations of hypergeometric functions. Consider the following situation. Suppose that $\Gamma_{1}<\Gamma_{2}$ are two arithmetic triangle groups with Hauptmoduls $z_{1}$ and $z_{2}$, respectively. Since any automorphic function on $\Gamma_{2}$ is also an automorphic function on $\Gamma_{1}$, we have $z_{2}=S\left(z_{1}\right)$ for some $S(x) \in \mathbb{C}(x)$. Likewise, if $f_{1}$ and $f_{2}$ are two automorphic forms of the same weight $k$ on $\Gamma_{1}$ and $\Gamma_{2}$, respectively, then the ratio $f_{1} / f_{2}$ is an automorphic function on $\Gamma_{1}$ and hence is equal to $R\left(z_{1}\right)$ for some $R(x) \in \mathbb{C}(x)$. In view of Proposition 4, after taking the $k$ th roots of the two sides of $f_{1} / f_{2}=R\left(z_{1}\right)$, we obtain an algebraic transformation of a hypergeometric function. This explains the existence of Kummer's, Goursat's and Vidūnas' transformations. (Of course, the triangle groups appearing in their transformations may not be arithmetic, but the argument above is still valid.)

More generally, assume that $\Gamma_{1}$ and $\Gamma_{2}$ are two commensurable arithmetic triangle groups such that the Shimura curve associated to $\Gamma=\Gamma_{1} \cap \Gamma_{2}$ has genus 0 . Let $z$ be a Hauptmodul on $\Gamma$. Then each of $z_{1}$ and $z_{2}$ is a rational function of $z$. Similarly, the ratio $f_{1} / f_{2}$ is also a rational function of $z$. Again, Proposition 4 yields an algebraic transformation of the form

$$
{ }_{2} F_{1}\left(a_{1}, b_{1} ; c_{1} ; S_{1}(z)\right)=R(z){ }_{2} F_{1}\left(a_{2}, b_{2} ; c_{2} ; S_{2}(z)\right)
$$

for some rational functions $S_{1}(z)$ and $S_{2}(z)$ and some algebraic function $R(z)$. This is the theory behind (3) and other algebraic transformations given in the paper.

Definition 7. Let $S(z) \in \mathbb{C}(z)$ be a rational function. If the finite covering $\mathbb{P}^{1}(\mathbb{C}) \rightarrow \mathbb{P}^{1}(\mathbb{C})$ defined by $S: z \rightarrow S(z)$ is ramified at most at three points 0 , 1 , and $\infty$, then $S$ is called a Belyi function.

In practice, the Belyi functions $S_{1}(z)$ and $S_{2}(z)$ can be determined by the ramification data of the coverings of Shimura curves. The function $R(z)$ can be determined by Propositions 2 and 4 .

We now obtain algebraic transformations of hypergeometric functions using the above idea. Note that according to [8], arithmetic triangle groups fall into 19 commensurability classes. The first class on his list corresponds to classical modular curves. In this case, it is easier to use classical modular forms to derive identities. We will not discuss this case here. Identities arising from Classes II, V, and XII are special cases of a family of identities, and so are identities from Classes IV, VIII, XI, XIII, XV, and XVII. These cases will be treated in a later section. Here we first consider Class III in Section 3 and Class VI in Section 4 (There are no identities from Classes IX and XIX since these classes consist of a single group. Also, identities from Classes VII, XIV, XVI, and XVIII are just Kummer's quadratic transformations. For Class $\mathrm{X}$, there is only one pair of triangle groups whose intersection has genus 0 . The identities associated to this pair are just consequences of Kummer's quadratic transformation and Goursat's cubic transformation. Thus, we will not discuss this case in the paper.)

\section{Algebraic transformations associated to Class III}

According to 88, Takeuchi's Class III of commensurable arithmetic triangle groups has the following subgroup diagram. Here because all groups involved have 
genus zero, we omit the genus information in the signatures of the groups:

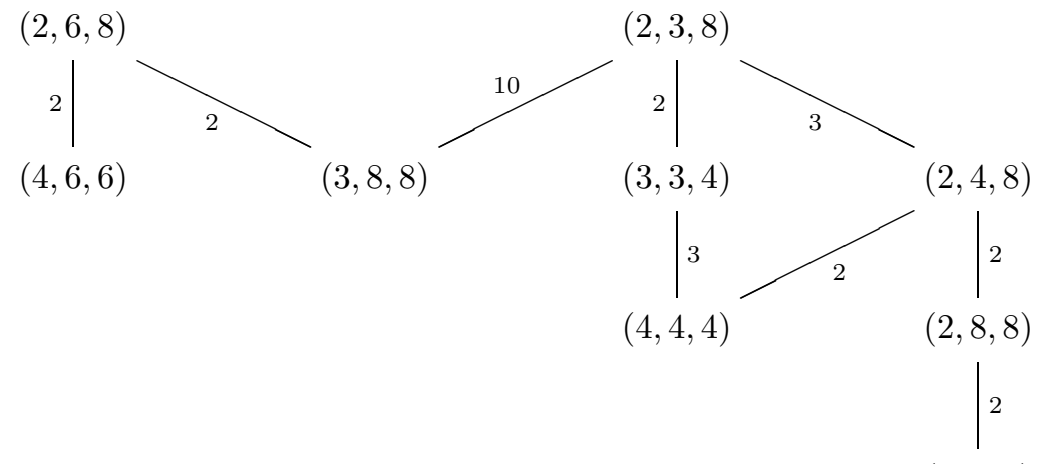

$(4,8,8)$

The main goal in this section is to prove an algebraic transformation associated to the pair of triangle groups $(4,6,6)$ and $(4,4,4)$.

Theorem 1. Let $\alpha$ be a root of $x^{2}+3=0$ and $\beta$ a root of $x^{2}+2=0$. We have

$$
\begin{array}{r}
\frac{(1+z)^{1 / 8}(1-3 z)^{1 / 8}}{(1+\alpha z)^{5 / 4}}{ }_{2} F_{1}\left(\frac{5}{24}, \frac{3}{8} ; \frac{3}{4} ; \frac{12 \alpha z\left(1-z^{2}\right)\left(1-9 z^{2}\right)}{(1+\alpha z)^{6}}\right) \\
=\frac{1}{\left(1+(4+2 \beta) z-(1+2 \beta) z^{2}\right)^{1 / 2}} 2 F_{1}\left(\frac{1}{8}, \frac{3}{8} ; \frac{3}{4} ; R(z)\right)
\end{array}
$$

and

(6)

$$
\begin{gathered}
\frac{(1-z)^{1 / 4}(1+z)^{5 / 8}(1-3 z)^{1 / 4}(1+3 z)^{5 / 8}}{(1+\alpha z)^{11 / 4}}{ }_{2} F_{1}\left(\frac{11}{24}, \frac{5}{8} ; \frac{5}{4} ; \frac{12 \alpha z\left(1-z^{2}\right)\left(1-9 z^{2}\right)}{(1+\alpha z)^{6}}\right) \\
=\frac{\left(1+(-7+4 \beta) z^{2} / 3\right)}{\left(1+(4+2 \beta) z-(1+2 \beta) z^{2}\right)^{3 / 2}} 2 F_{1}\left(\frac{3}{8}, \frac{5}{8} ; \frac{5}{4} ; R(z)\right)
\end{gathered}
$$

where

$$
R(z)=-\frac{4(1+\beta)^{4} z\left(1+(-7+4 \beta) z^{2} / 3\right)^{4}}{(1+z)(1-3 z)\left(1+(4+2 \beta) z-(1+2 \beta) z^{2}\right)^{4}} .
$$

We first determine the signatures of the intersections.

Lemma 8. We have

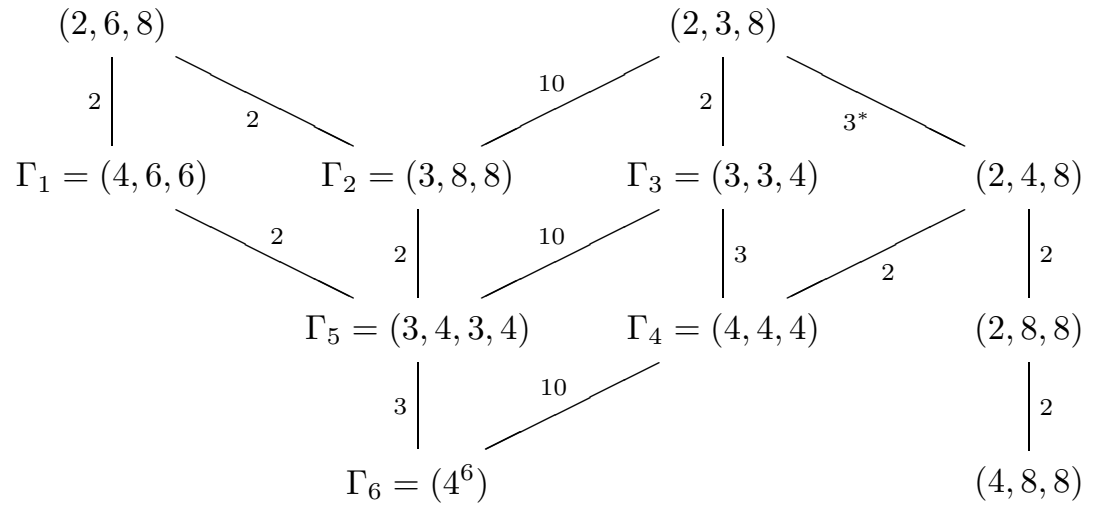


Moreover, the group of signature $\left(4^{6}\right)$ is a normal subgroup of the group of signature $(3,4,3,4)$. (Here $\left(4^{6}\right)$ is shorthand for $(4,4,4,4,4,4)$.)

Proof. Let $\Gamma_{2}=(3,8,8)$ and $\Gamma_{2}^{\prime}$ be its commutator subgroup. From the group presentation

$$
\Gamma_{2} \simeq\left\langle\gamma_{1}, \gamma_{2}: \gamma_{1}^{3}, \gamma_{2}^{8},\left(\gamma_{1} \gamma_{2}\right)^{8}\right\rangle
$$

for $\Gamma_{2}$, we know that $\Gamma_{2} / \Gamma_{2}^{\prime}$ is cyclic of order 8 . Thus, $\Gamma_{2}$ has exactly one subgroup of index 2, which must be the common intersection of the groups $(4,6,6),(3,8,8)$ and $(3,3,4)$. The signature of this subgroup can be easily determined by observing that a covering of degree 2 from a Shimura curve to the Shimura curve associated to $(3,8,8)$ can only ramify at the two elliptic points of order 8 . We find that the signature must be $(3,4,3,4)$.

We next observe that the commutator subgroup $\Gamma_{3}^{\prime}$ of the group $\Gamma_{3}=(3,3,4)$ is a normal subgroup of index 3 in $\Gamma_{3}$. We claim that this $\Gamma_{3}^{\prime}$ must be the same as the group of signature $(4,4,4)$. If $\Gamma_{3}^{\prime} \neq(4,4,4)$, then $\Gamma_{3}^{\prime} \cap(4,4,4)$ is a normal subgroup of $(4,4,4)$ of index 3 , but the group $(4,4,4)$ cannot have a normal subgroup of index 3. We next determine the signature of the intersection of $\Gamma_{4}=(4,4,4)$ and $\Gamma_{5}=(3,4,3,4)$.

Let $X_{j}$ denote the Shimura curve associated to the group $\Gamma_{j}$. Since $\Gamma_{4}$ is a normal subgroup of $\Gamma_{3}$ of index 3 , the intersection $\Gamma_{6}$ of $\Gamma_{4}$ and $\Gamma_{5}$ is a normal subgroup of index 3 in $\Gamma_{5}$, which implies that the two elliptic points of order 4 of $X_{5}$ must split completely on $X_{6}$. In view of the Riemann-Hurwitz formula, the two elliptic points of order 3 of $X_{5}$ must be totally ramified. We conclude that $\Gamma_{6}$ has signature $\left(4^{6}\right)$.

In fact, the subgroup relations mentioned above can be visualized by the following figures.
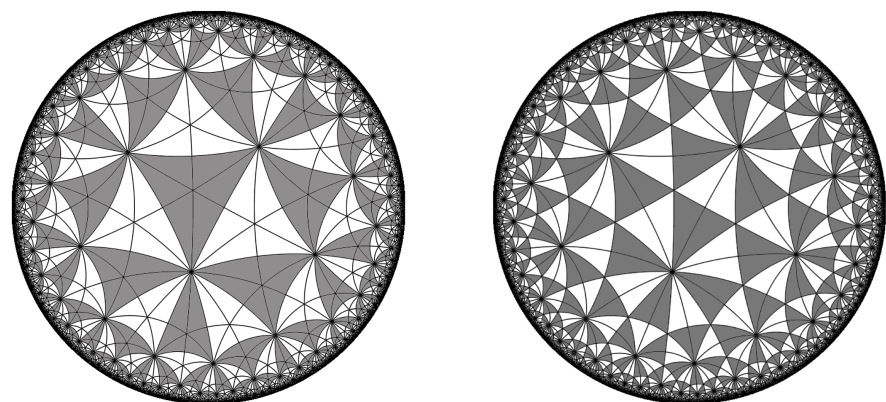

Here the small triangles are $(2,3,8)$-triangles. Let $G$ be the group of all symmetries of the tessellation of the hyperbolic plane by the $(4,4,4)$-triangles and $G_{0}$ be the subgroup generated by the reflections across the edges of the $(4,4,4)$-triangles. Then $G / G_{0}$ is isomorphic to $D_{3}$. The $(3,3,4)$-triangle group corresponds to the cyclic subgroup of order 3 in $G / G_{0}$, while the group $(2,3,8)$ corresponds to the whole group $G / G_{0}$. Similarly, if we piece 12 copies of the $(2,6,8)$-triangles around the vertex of inner angle $\pi / 4$, we get a regular hexagon with inner angles $\pi / 4$. Let $H$ be the group of all symmetries of the tessellation by this regular hexagon and $H_{0}$ be the subgroup generated by the reflections across the edges of hexagons. Then $H / H_{0}$ is isomorphic to $D_{6}$. The unique cyclic subgroup of order 3 in $H / H_{0}$ corresponds to the group $(3,4,3,4)$. See the figures below. 

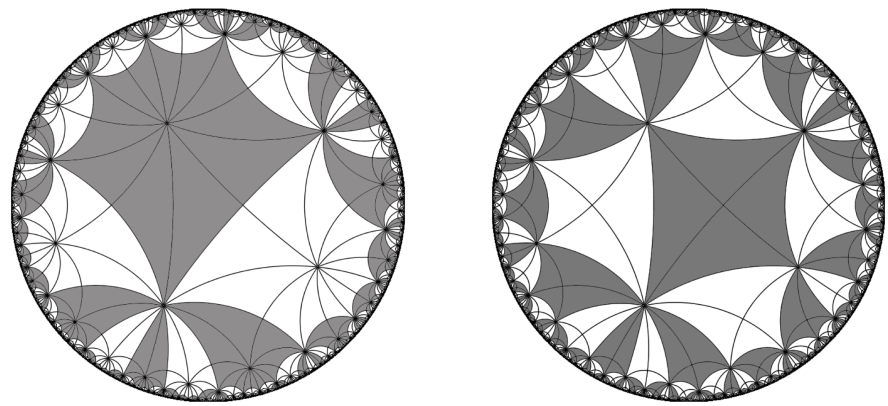

(The groups $(2,6,8),(4,6,6)$, and $(3,8,8)$ correspond to the whole $H / H_{0}$, the cyclic subgroup of order 6 of $H / H_{0}$, and one of the $D_{3}$-subgroups, respectively.)

Now let $\Gamma_{1}=(4,6,6), \Gamma_{2}=(3,8,8), \Gamma_{3}=(3,3,4), \Gamma_{4}=(4,4,4), \Gamma_{5}=(3,4,3,4)$, and $\Gamma_{6}=\left(4^{6}\right)$. Let $X_{j}=X\left(\Gamma_{j}\right), j=1, \ldots, 6$, be the corresponding Shimura curves. Label the elliptic points on $X_{1}$ by $P_{4}, P_{6}$, and $P_{6}^{\prime}$, those on $X_{2}$ by $Q_{3}, Q_{8}$, and $Q_{8}^{\prime}$, those on $X_{3}$ by $R_{3}, R_{3}^{\prime}$, and $R_{4}$, those on $X_{4}$ by $S_{4}, S_{4}^{\prime}, S_{4}^{\prime \prime}$, and those on $X_{5}$ by $T_{3}, T_{3}^{\prime}, T_{4}$, and $T_{4}^{\prime}$ (with the subscripts carrying the obvious meaning) such that the ramification data are given by
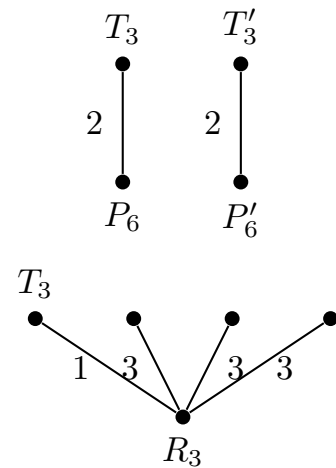
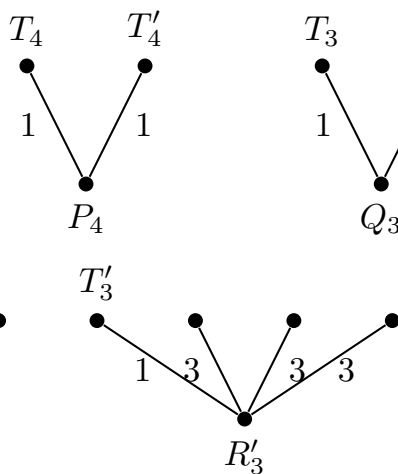
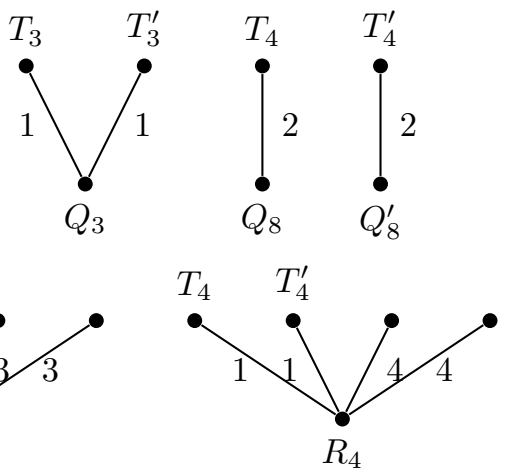

Label the elliptic points of $X_{6}$ by $U_{1}, \ldots, U_{6}$ such that the rotation around the center of the $\left(4^{6}\right)$-polygon by the angle $\pi / 3$ permutes the six points cyclically. From the figures above, we know that if we label the points such that $U_{1}$ lies above $T_{4}$, then the ramification data for $X_{6} \rightarrow X_{5}$ are
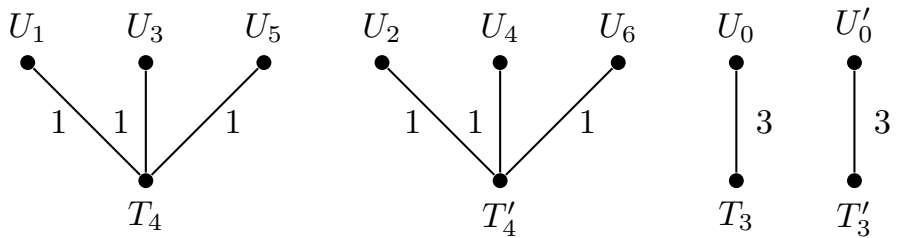

where $U_{0}$ and $U_{0}^{\prime}$ are the centers of the $\left(4^{6}\right)$-polygons. (The reader is reminded that each $\left(4^{6}\right)$-polygon represents only half of the fundamental domain for the Shimura curve $X_{6}$. Referring to the figure in the proof of the lemma above, a fundamental domain consists of a grey $\left(4^{6}\right)$-polygon and a neighboring white $\left(4^{6}\right)$-polygon.)

Lemma 9. The two elliptic points of $X_{6}$ at the two ends of a diagonal of a $\left(4^{6}\right)$ polygon lie above the same elliptic point of $X_{4}$. That is, labeling the elliptic points 
of $X_{4}$ suitably, we have
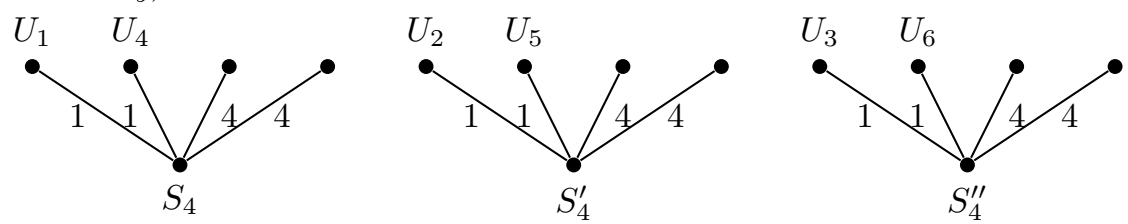

Moreover, if we choose Hauptmoduls $z_{j}(\tau)$ for $X_{j}, j=1, \ldots, 6$, by requiring

$$
\begin{aligned}
& z_{1}\left(P_{4}\right)=0, \quad z_{1}\left(P_{6}\right)=1, \quad z_{1}\left(P_{6}^{\prime}\right)=\infty, \\
& z_{2}\left(Q_{8}\right)=0, \quad z_{2}\left(Q_{3}\right)=1, \quad z_{2}\left(Q_{8}^{\prime}\right)=\infty, \\
& z_{3}\left(R_{4}\right)=0, \quad z_{3}\left(R_{3}\right)=1, \quad z_{3}\left(R_{3}^{\prime}\right)=\infty, \\
& z_{4}\left(S_{4}\right)=0, \quad z_{4}\left(S_{4}^{\prime}\right)=1, \quad z_{4}\left(S_{4}^{\prime \prime}\right)=\infty, \\
& z_{5}\left(T_{4}\right)=0, \quad z_{5}\left(T_{3}\right)=1, \quad z_{5}\left(T_{4}^{\prime}\right)=\infty, \\
& z_{6}\left(U_{1}\right)=0, \quad z_{6}\left(U_{3}\right)=1, \quad z_{6}\left(U_{4}\right)=\infty,
\end{aligned}
$$

then we have

$$
\begin{gathered}
z_{1}=\frac{4 z_{5}}{\left(1+z_{5}\right)^{2}}, \quad z_{2}=z_{5}^{2} \\
z_{3}=\frac{3\left(\zeta-\zeta^{2}\right) z_{4}\left(1-z_{4}\right)}{\left(1+\zeta z_{4}\right)^{3}}, \quad z_{5}=\frac{3\left(\zeta-\zeta^{2}\right) z_{6}\left(1-z_{6}^{2}\right)}{1-9 z_{6}^{2}}, \\
z_{3}=\frac{(28+16 \beta) z_{5}\left(1+(-17+56 \beta) z_{5}^{2} / 81\right)^{4}}{\left(1+z_{5}\right)\left(1+(13+8 \beta) z_{5} / 3-(25+32 \beta) z_{5}^{2} / 9+(17-56 \beta) z_{5}^{3} / 81\right)^{3}},
\end{gathered}
$$

and

$$
z_{4}=-\frac{4(1+\beta)^{4} z_{6}\left(1+(-7+4 \beta) z_{6}^{2} / 3\right)^{4}}{\left(1+z_{6}\right)\left(1-3 z_{6}\right)\left(1+(4+2 \beta) z_{6}-(1+2 \beta) z_{6}^{2}\right)^{4}},
$$

where $\zeta$ is a 3 rd root of unity and $\beta$ is a root of $x^{2}+2=0$.

Proof. The ramification data for the covering $X_{5} \rightarrow X_{2}$ and the assumption $z_{2}\left(Q_{3}\right)$ $=z_{5}\left(T_{3}\right)=1$ imply that $z_{2}=z_{5}^{2}$ and

$$
z\left(T_{3}^{\prime}\right)=-1
$$

The relation between $z_{1}$ and $z_{5}$ is easy to determine. We find $z_{1}=4 z_{5} /\left(1+z_{5}\right)^{2}$.

To determine the relation between $z_{3}$ and $z_{4}$, we recall from Lemma 8 that $\Gamma_{4}$ is a normal subgroup of $\Gamma_{3}$. Any element of $\Gamma_{3}$ not in $\Gamma_{4}$ induces an automorphism of order 3 on $X_{4}$. Such an automorphism must permute the three elliptic points $S_{4}, S_{4}^{\prime}$, and $S_{4}^{\prime \prime}$ cyclically. In terms of the Hauptmodul $z_{4}$, such an automorphism is either

$$
\sigma: z_{4} \longmapsto \frac{-1}{z_{4}-1}
$$

or its square. Moreover, the fixed points of such an automorphism are the ramified points in the covering $X_{4} \rightarrow X_{3}$. That is, if we let $S_{0}$ and $S_{0}^{\prime}$ be the points lying above $R_{3}$ and $R_{3}^{\prime}$ respectively, then $z_{4}\left(S_{0}\right), z_{4}\left(S_{0}^{\prime}\right) \in\left\{-\zeta,-\zeta^{2}\right\}$, where $\zeta$ is a primitive 3 rd root of unity. Then from the ramification data, we easily deduce that $z_{3}=\left(\zeta-\zeta^{2}\right) z_{4}\left(1-z_{4}^{2}\right) /\left(1+\zeta z_{4}\right)^{3}$.

To determine the relation between $z_{5}$ and $z_{6}$, we argue similarly as above. The tessellation of the hyperbolic plane by $\Gamma_{6}$ has a $D_{6}$-symmetry, in addition to the symmetries arising from the reflections across the edges of the $\left(4^{6}\right)$-polygons. This 
provides much useful information. For example, if we let $\tau$ be the reflection across the diagonal joining $U_{1}$ and $U_{4}$, then $\tau$ induces an involution on $X_{6}$, which, in terms of $z_{6}$, is given by

$$
\tau: z_{6} \longmapsto-z_{6},
$$

which implies that

$$
z_{6}\left(U_{5}\right)=-1 \text {. }
$$

Furthermore, let $\rho$ denote the rotation by angle $\pi / 3$ around the center of the hexagon. Then

$$
\rho: z_{6} \longmapsto \frac{c z_{6}+1}{-c z_{6}+c}
$$

for some zero constant $c$ since $\rho$ maps 1 to $\infty$ and $\infty$ to -1 . In light of $\rho^{2}: 0 \rightarrow 1$, we conclude that $c=3$ and

$$
z_{6}\left(U_{2}\right)=1 / 3, \quad z_{6}\left(U_{6}\right)=-1 / 3 .
$$

It follows that $z_{5}=A z_{6}\left(1-z_{6}^{2}\right) /\left(1-9 z_{6}^{2}\right)$ for some $A$. This constant $A$ has the property that $A x\left(1-x^{2}\right)-\left(1-9 x^{2}\right)$ has repeated roots. We find $A= \pm 3 \sqrt{-3}$. The choice of the sign must be synchronized with the choice of the third root of unity in the relation between $z_{4}$ and $z_{5}$. This will be done later.

We now come to the more complicated part of the lemma. Let $\pi: X_{6} \rightarrow X_{4}$ be the covering of the Shimura curves. Let $\gamma$ be an element of $\Gamma_{5}$ not in $\Gamma_{6}$. Then $\gamma$ normalizes both $\Gamma_{4}$ and $\Gamma_{6}$ and induces automorphisms $\rho_{1}$ and $\rho_{2}$ on $X_{4}$ and $X_{6}$, respectively. We may assume that $\rho_{2}=\rho^{2}$, where $\rho$ permutes $U_{1}, \ldots, U_{6}$ cyclically, as defined in the previous paragraph. It is easy to check that $\pi \circ \rho_{1}=\rho_{2} \circ \pi$. Thus, $\pi\left(U_{1}\right), \pi\left(U_{3}\right)$, and $\pi\left(U_{5}\right)$ are three different elliptic points on $X_{4}$. We label them by $S_{4}, S_{4}^{\prime}$, and $S_{4}^{\prime \prime}$, respectively. Let $V_{1}, V_{2}$ be the two ramified points lying above $S_{4}$. Now there are three possibilities:

$\pi^{-1}\left(S_{4}\right)=\left\{U_{1}, U_{2}, V_{1}, V_{2}\right\}, \pi^{-1}\left(S_{4}\right)=\left\{U_{1}, U_{4}, V_{1}, V_{2}\right\}, \pi^{-1}\left(S_{4}\right)=\left\{U_{1}, U_{6}, V_{1}, V_{2}\right\}$.

We will show that the correct one is $\left\{U_{1}, U_{4}, V_{1}, V_{2}\right\}$.

Let $V_{j}^{\prime}=\rho_{2}\left(V_{j}\right)$ and $V_{j}^{\prime \prime}=\rho_{2}^{2}\left(V_{j}\right)$ for $j=1,2$. If $\pi^{-1}\left(S_{4}\right)=\left\{U_{1}, U_{2}, V_{1}, V_{2}\right\}$, then we have

$$
z_{4}=\frac{B z_{6}\left(1-3 z_{6}\right)\left(z_{6}-z_{6}\left(V_{1}\right)\right)^{4}\left(z_{6}-z_{6}\left(V_{2}\right)\right)^{4}}{\left(1+z_{6}\right)\left(1+3 z_{6}\right)\left(z_{6}-z_{6}\left(V_{1}^{\prime \prime}\right)\right)^{4}\left(z_{6}-z_{6}\left(V_{2}^{\prime \prime}\right)\right)^{4}}
$$

for some constant $B$. The values of $z_{6}\left(V_{1}\right)$, etc. must satisfy

$$
\begin{aligned}
& B x(1-3 x)\left(1-x / z_{6}\left(V_{1}\right)\right)^{4}\left(1-x / z_{6}\left(V_{2}\right)\right)^{4} \\
&-(1+x)(1+3 x)\left(1-x / z_{6}\left(V_{1}^{\prime \prime}\right)\right)^{4}\left(1-x / z_{6}\left(V_{2}^{\prime \prime}\right)\right)^{4} \\
&=C(1-x)\left(1-x / z_{6}\left(V_{1}^{\prime}\right)\right)^{4}\left(1-x / z_{6}\left(V_{2}^{\prime}\right)\right)^{4}
\end{aligned}
$$

for some constant $C$. Now if we let $p_{1}(x)=1+a x+b x^{2}=\left(1-x / z_{6}\left(V_{1}\right)\right)(1-$ $\left.x / z_{6}\left(V_{2}\right)\right)$, then $\left(1-x / z_{6}\left(V_{1}^{\prime}\right)\right)\left(1-x / z_{6}\left(V_{2}^{\prime}\right)\right)$ and $\left(1-x / z_{6}\left(V_{1}^{\prime \prime}\right)\left(1-x / z_{6}\left(V_{2}^{\prime \prime}\right)\right)\right.$ are scalar multiples of

$$
\begin{aligned}
& p_{2}(x)=(1+3 x)^{2} p_{1}\left(\frac{x-1}{3 x+1}\right)=(1-a+b)+(6-2 a-2 b) x+(9+3 a+b) x^{2}, \\
& p_{3}(x)=(1-3 x)^{2} p_{1}\left(\frac{x+1}{1-3 x}\right)=(1+a+b)+(-6-2 a+2 b) x+(9-3 a+b) x^{2},
\end{aligned}
$$


respectively. Substituting these into (17) and equating the coefficients in the two sides, we find $B=C=0, a=2, b=-3$, but obviously this is invalid. This means that $\pi^{-1}\left(S_{4}\right) \neq\left\{U_{1}, U_{2}, V_{1}, V_{2}\right\}$. Likewise, $\pi^{-1}\left(S_{4}\right) \neq\left\{U_{1}, U_{6}, V_{1}, V_{2}\right\}$. Thus, we must have $\pi^{-1}\left(S_{4}\right)=\left\{U_{1}, U_{4}, V_{1}, V_{2}\right\}$. Now equating the coefficients in the two sides of

$$
B x\left(1+a x+b x^{2}\right)^{4}-(1-x)(1+3 x) p_{2}(x)^{4}=C(1+x)(1-3 x) p_{3}(x)^{4}
$$

and excluding the invalid solutions, we get the claimed relation between $z_{4}$ and $z_{6}$. The relation between $z_{3}$ and $z_{5}$ can be determined by the known relation between $z_{3}$ and $z_{4}$, that between $z_{4}$ and $z_{6}$, and that between $z_{5}$ and $z_{6}$. This process also determines the choices of the third roots of unity in the relation between $z_{3}$ and $z_{4}$ and that between $z_{5}$ and $z_{6}$. We omit the details.

Lemma 10. The automorphic derivative $Q\left(z_{6}\right)=D\left(z_{6}, \tau\right)$ is equal to

$$
\begin{aligned}
\frac{15}{64}\left(\frac{1}{z_{6}^{2}}+\right. & \left.\frac{1}{\left(1-z_{6}\right)^{2}}+\frac{1}{\left(1+z_{6}\right)^{2}}+\frac{1}{\left(z_{6}-1 / 3\right)^{2}}+\frac{1}{\left(z_{6}+1 / 3\right)^{2}}\right) \\
& +\frac{45}{128}\left(\frac{1}{1-z_{6}}+\frac{1}{1+z_{6}}+\frac{3}{1-3 z_{6}}+\frac{3}{1+3 z_{6}}\right) .
\end{aligned}
$$

Proof. By Proposition 2, the rational function $R(x)$ such that automorphic $Q\left(z_{6}\right)=$ $D\left(z_{6}, \tau\right)$ is equal to $R\left(z_{6}\right)$ is equal to

$$
\begin{gathered}
R(x)=\frac{15}{64}\left(\frac{1}{x^{2}}+\frac{1}{(1-x)^{2}}+\frac{1}{(1+x)^{2}}+\frac{1}{(x-1 / 3)^{2}}+\frac{1}{(x+1 / 3)^{2}}\right) \\
+\frac{B_{1}}{x}+\frac{B_{2}}{x-1}+\frac{B_{3}}{x+1}+\frac{B_{4}}{x-1 / 3}+\frac{B_{5}}{x+1 / 3}
\end{gathered}
$$

for some constants $B_{j}$ satisfying

$$
B_{1}+B_{2}+B_{3}+B_{4}+B_{5}=0, \quad B_{2}-B_{3}+\frac{1}{3} B_{4}-\frac{1}{3} B_{5}+\frac{15}{16}=0 .
$$

Now the normalizer of $\Gamma_{6}$ in $\operatorname{SL}(2, \mathbb{R})$ contains at least the group of signature $(2,6,8)$. The factor group, in terms of the Hauptmodul $z_{6}$, is generated by $\sigma$ : $z_{6} \mapsto\left(3 z_{6}+1\right) /\left(-3 z_{6}+3\right)$ and $\tau: z_{6} \mapsto-z_{6}$. By Proposition [6, $R(x)$ satisfies

$$
R(-x)=R(x), \quad \frac{144}{(-3 x+3)^{4}} R\left(\frac{3 x+1}{-3 x+3}\right)=R(x) .
$$

Combining this information with (9), we find

$$
B_{1}=0, \quad B_{2}=B_{4}=-\frac{45}{128}, \quad B_{3}=B_{5}=\frac{45}{128} .
$$

This gives us the formula.

We now prove the theorem.

Proof of Theorem 1. By Proposition 1, we have

$$
\begin{aligned}
& \operatorname{dim} S_{6}\left(\Gamma_{1}\right)=1-6+\left\lfloor\frac{6}{2}\left(1-\frac{1}{4}\right)\right\rfloor+2\left\lfloor\frac{6}{2}\left(1-\frac{1}{6}\right)\right\rfloor=1, \\
& \operatorname{dim} S_{6}\left(\Gamma_{4}\right)=1-6+3\left\lfloor\frac{6}{2}\left(1-\frac{1}{4}\right)\right\rfloor=1, \\
& \operatorname{dim} S_{6}\left(\Gamma_{6}\right)=1-6+6\left\lfloor\frac{6}{2}\left(1-\frac{1}{4}\right)\right\rfloor=7 .
\end{aligned}
$$


By Proposition 4, the one-dimensional spaces $S_{6}\left(\Gamma_{1}\right)$ and $S_{6}\left(\Gamma_{4}\right)$ are spanned by

$$
F_{1}=z_{1}^{1 / 4}\left(1-z_{1}\right)^{1 / 2}\left({ }_{2} F_{1}\left(\frac{5}{24}, \frac{3}{8} ; \frac{3}{4} ; z_{1}\right)+C_{1} z_{1}^{1 / 4}{ }_{2} F_{1}\left(\frac{11}{24}, \frac{5}{8} ; \frac{5}{4} ; z_{1}\right)\right)^{6}
$$

and

$$
F_{2}=z_{4}^{1 / 4}\left(1-z_{4}\right)^{1 / 4}\left({ }_{2} F_{1}\left(\frac{1}{8}, \frac{3}{8} ; \frac{3}{4} ; z_{4}\right)+C_{2} z_{4}^{1 / 4}{ }_{2} F_{1}\left(\frac{3}{8}, \frac{5}{8} ; \frac{5}{4} ; z_{4}\right)\right)^{6}
$$

for some complex numbers $C_{1}$ and $C_{2}$, respectively. Furthermore, by Proposition 2 if we let

$$
\begin{aligned}
& f_{1}=z_{6}^{3 / 8}\left(1-\frac{15}{7} z_{6}^{2}-\frac{111}{14} z_{6}^{4}-\frac{2045}{46} z_{6}^{6}-\frac{11355195}{39928} z_{6}^{8}-\frac{77997477}{39928} z_{6}^{10}-\cdots\right) \\
& f_{2}=z_{6}^{5 / 8}\left(1-\frac{5}{3} z_{6}^{2}-\frac{245}{34} z_{6}^{4}-\frac{7269}{170} z_{6}^{6}-\frac{115223}{408} z_{6}^{8}-\frac{55230121}{27880}-\cdots\right)
\end{aligned}
$$

be a basis for the solution space of the Schwarzian differential equation $d^{2} f / d z_{6}^{2}+$ $Q\left(z_{6}\right) f=0$, where $Q\left(z_{6}\right)$ is the rational function in (8), then a basis for $S_{6}\left(\Gamma_{6}\right)$ is

$$
\left\{z_{6}^{j} g: j=0, \ldots, 6\right\}, \quad g=\frac{\left(f_{1}+C_{3} f_{2}\right)^{6}}{z_{6}^{2}\left(1-z_{6}^{2}\right)^{2}\left(1-9 z_{6}^{2}\right)^{2}} .
$$

Now from Lemma 9] we have

$$
z_{1}=\frac{12 \alpha z_{6}\left(1-z_{6}^{2}\right)\left(1-9 z_{6}^{2}\right)}{\left(1+\alpha z_{6}\right)^{6}}
$$

and

$$
z_{4}=-\frac{4(1+\beta)^{4} z_{6}\left(1+(-7+4 \beta) z_{6}^{2} / 3\right)^{4}}{\left(1+z_{6}\right)\left(1-3 z_{6}\right)\left(1+(4+2 \beta) z_{6}-(1+2 \beta) z_{6}^{2}\right)^{4}}
$$

where $\alpha$ is a root of $x^{2}+3=0$ and $\beta$ is a root of $x^{2}+2=0$. Substituting these into (10) and (11) and comparing the coefficients, we find

$$
F_{1}=c_{1}\left(1+3 z_{6}^{2}\right)^{3} g
$$

and

$$
\begin{aligned}
F_{2}=c_{2}(1+ & \left.\frac{-7+4 \beta}{3} z_{6}^{2}\right)\left(1+(4+2 \beta) z_{6}-(1+2 \beta) z_{6}^{2}\right) \\
& \times\left(1-(4+2 \beta) z_{6}-(1+2 \beta) z_{6}^{2}\right) g
\end{aligned}
$$

for some constants $c_{1}$ and $c_{2}$. Taking the sixth roots of $F_{1}$ and $F_{2}$ and simplifying, we obtain the identities claimed in the theorem. 


\section{Algebraic transformations associated to Class Vi}

According to Appendix A, the subgroup diagram for Takeuchi's Class VI is

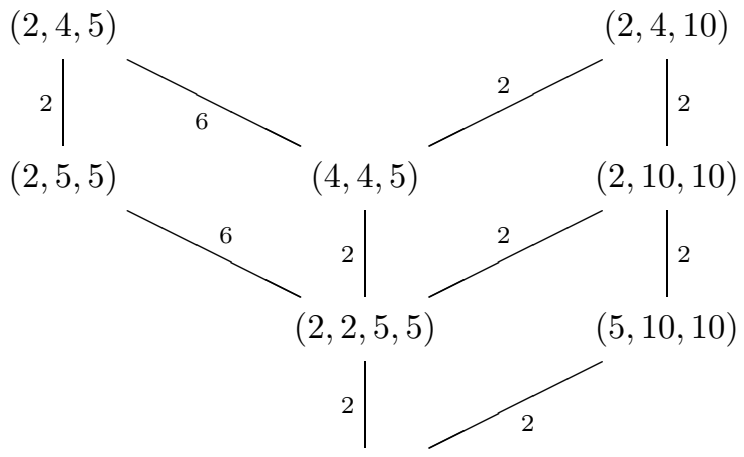

$(5,5,5,5)$

Let $\Gamma_{1}=(2,5,5), \Gamma_{2}=(5,10,10), \Gamma_{3}=(5,5,5,5)$, and $X_{1}, X_{2}, X_{3}$ be the Shimura curves associated to these three groups. (The reader is reminded that the subgroup diagram should be read as "there are arithmetic Fuchsian subgroups of $\operatorname{SL}(2, \mathbb{R})$ such that their subgroup relations are given by the diagram".) The subgroup relations $\Gamma_{3}<\Gamma_{1}, \Gamma_{2}$ admit Coxeter decompositions as the following figures show:
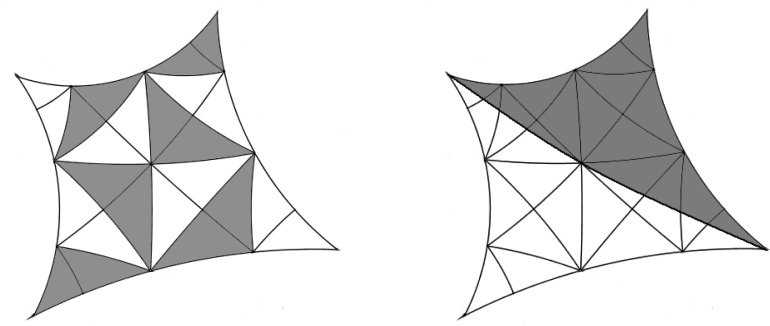

Here the small triangles are $(2,4,5)$-triangles. Associated to this triplet of groups are the following identities.

Theorem 2. We have

$$
\begin{aligned}
{ }_{2} F_{1}\left(\frac{1}{20},\right. & \left.\frac{1}{4} ; \frac{4}{5} ; \frac{64 z\left(1-z-z^{2}\right)^{5}}{\left(1-z^{2}\right)\left(1+4 z-z^{2}\right)^{5}}\right) \\
& =\left(1-z^{2}\right)^{1 / 20}\left(1+4 z-z^{2}\right)^{1 / 4}{ }_{2} F_{1}\left(\frac{3}{10}, \frac{2}{5} ; \frac{9}{10} ; z^{2}\right)
\end{aligned}
$$

and

$$
\begin{aligned}
& \left(1-z-z^{2}\right)_{2} F_{1}\left(\frac{1}{4}, \frac{9}{20} ; \frac{6}{5} ; \frac{64 z\left(1-z-z^{2}\right)^{5}}{\left(1-z^{2}\right)\left(1+4 z-z^{2}\right)^{5}}\right) \\
& =\left(1-z^{2}\right)^{1 / 4}\left(1+4 z-z^{2}\right)^{5 / 4}{ }_{2} F_{1}\left(\frac{2}{5}, \frac{1}{2} ; \frac{11}{10} ; z^{2}\right) .
\end{aligned}
$$


Proof. Label the elliptic points of $X_{j}$ by $P_{2}, P_{5}, P_{5}^{\prime}$ for $X_{1}, Q_{5}, Q_{10}, Q_{10}^{\prime}$ for $X_{2}$, and $R_{i}, i=1, \ldots, 4$, for $X_{3}$ such that the ramifications data are given by
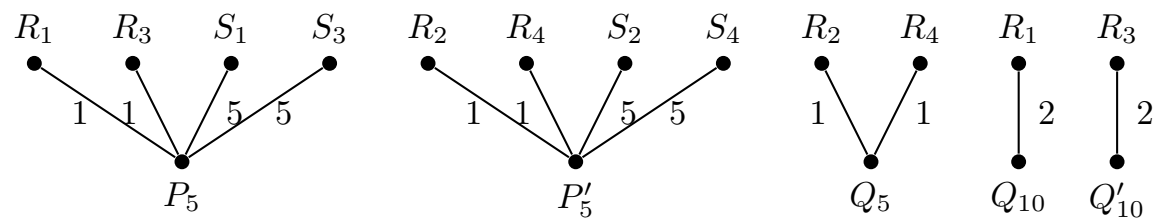

Here the numbers next to the lines are the ramification indices. We have omitted $P_{2}$ from the diagram. There are 6 points lying above $P_{2}$. Each has ramficiation index 2. Choose Hauptmoduls $z_{j}$ for $X_{j}$ by requiring

$$
z_{1}\left(P_{5}\right)=0, z_{1}\left(P_{2}\right)=1, z_{1}\left(P_{5}^{\prime}\right)=\infty, \quad z_{2}\left(Q_{10}\right)=0, z_{2}\left(Q_{5}\right)=1, z_{2}\left(Q_{10}^{\prime}\right)=\infty
$$

and

$$
z_{3}\left(R_{1}\right)=0, z_{3}\left(R_{2}\right)=1, z_{3}\left(R_{3}\right)=\infty .
$$

The relation between $z_{2}$ and $z_{3}$ is easy to figure out. We have

$$
z_{2}=z_{3}^{2}
$$

which implies that $z_{3}\left(R_{4}\right)=-1$. To determine the relation between $z_{1}$ and $z_{3}$, we observe that the tessellation of the hyperbolic plane by the $(5,5,5,5)$-polygons has extra symmetries by rotation by 90 degrees around the center of any $(5,5,5,5)$ polygon. In terms of groups, this means that $\Gamma_{3}$ has a supergroup $\Gamma$ normalizing $\Gamma_{3}$ such that $\Gamma / \Gamma_{3}$ is cyclic of order 4 . (In fact, $\Gamma$ is the $(4,4,5)$-triangle group in the subgroup diagram.) Therefore, the automorphism group of $X_{3}$ has an element $\sigma$ of order 4 that permutes $R_{1}, R_{2}, R_{3}, R_{4}$ cyclically. In terms of the Hauptmodul, we have

$$
\sigma: z_{3} \longmapsto \frac{z_{3}-1}{z_{3}+1}
$$

Thus, if the value of $z_{3}$ at $S_{1}$ is $a$, then we have

$$
z_{3}\left(S_{1}\right)=a, \quad z_{3}\left(S_{2}\right)=\frac{a-1}{a+1}, \quad z_{3}\left(S_{3}\right)=-\frac{1}{a}, \quad z_{3}\left(S_{4}\right)=-\frac{a+1}{a-1} .
$$

Therefore, the relation between $z_{1}$ and $z_{3}$ is

$$
z_{1}=\frac{B z_{3}\left(z_{3}-a\right)^{5}\left(z_{3}+1 / a\right)^{5}}{\left(1-z_{3}^{2}\right)\left(z_{3}-(a-1) /(a+1)\right)^{5}\left(z_{3}+(a+1) /(a-1)\right)^{5}}
$$

for some constant $B$. Moreover, the automorphism $\sigma$ of $X_{3}$ rotates four of the six points lying above $P_{2}$ cyclically and fixes the other two. (The reader is reminded that each $(5,5,5,5)$-polygon represents only half of the fundamental domain for $\Gamma_{3}$. The two fixed points of $\sigma$ are the centers of the (5,5,5,5)-polygons.) In terms of the Hauptmodul $z_{3}$, this means that the values of $z_{3}$ at the two fixed points of $\sigma$ are $\pm i$ and if the value of $z_{3}$ at one of the other four points above $P_{2}$ is $b$, then the values at the other three points are $-1 / b,(b-1) /(b+1)$, and $-(b+1) /(b-1)$. Thus, we have

$$
z_{1}-1-1=\frac{C\left(1+z_{3}^{2}\right)^{2}\left(z_{3}-b\right)^{2}\left(z_{3}+1 / b\right)^{2}\left(z_{3}-(b-1) /(b+1)\right)^{2}\left(z_{3}+(b+1) /(b-1)\right)^{2}}{\left(1-z_{3}^{2}\right)\left(z_{3}-(a-1) /(a+1)\right)^{5}\left(z_{3}+(a+1) /(a-1)\right)^{5}}
$$


for some constant $C$. Comparing the two sides, we find $a=0, \pm 1, \pm i, a^{2}+a-1=0$, or $a^{2}-a-1=0$. The first five solutions are invalid. The other two solutions give

$$
z_{1}=\frac{64 z_{3}\left(1-z_{3}-z_{3}^{2}\right)^{5}}{\left(1-z_{3}^{2}\right)\left(1+4 z_{3}-z_{3}^{2}\right)^{5}}
$$

or

$$
z_{1}=-\frac{64 z_{3}\left(1+z_{3}-z_{3}^{2}\right)^{5}}{\left(1-z_{3}^{2}\right)\left(1-4 z_{3}-z_{3}^{2}\right)^{5}}
$$

Both are valid because of the following reason. Notice that $\Gamma_{2}$ normalizes $\Gamma_{3}$. If we take an element $\gamma$ of $\Gamma_{2}$ not in $\Gamma_{3}$, then $\gamma^{-1} \Gamma_{1} \gamma$ is again a triangle of signature $(2,5,5)$ containing the same $\Gamma_{3}$. If the relation between the Hauptmoduls of $\Gamma_{1}$ and $\Gamma_{3}$ is (15), then the relation between the Hauptmoduls of $\gamma^{-1} \Gamma_{1} \gamma$ and $\Gamma_{3}$ will be (16).

Having determined the relations among Hauptmoduls, we can composite identity (36) in [10] with Kummer's quadratic transformation several times to get the identities in the theorem. However, the procedure is very tedious. Here we provide a better proof using the theory of automorphic forms on Shimura curves.

By Proposition 1, we have

$$
\begin{aligned}
& \operatorname{dim} S_{8}\left(\Gamma_{1}\right)=1-8+\left\lfloor\frac{8}{2}\left(1-\frac{1}{2}\right)\right\rfloor+2\left\lfloor\frac{8}{2}\left(1-\frac{1}{5}\right)\right\rfloor=1, \\
& \operatorname{dim} S_{8}\left(\Gamma_{2}\right)=1-8+\left\lfloor\frac{8}{2}\left(1-\frac{1}{5}\right)\right\rfloor+2\left\lfloor\frac{8}{2}\left(1-\frac{1}{10}\right)\right\rfloor=2, \\
& \operatorname{dim} S_{8}\left(\Gamma_{3}\right)=1-8+4\left\lfloor\frac{8}{2}\left(1-\frac{1}{5}\right)\right\rfloor=5 .
\end{aligned}
$$

By Proposition 4, the one-dimensional space $S_{8}\left(\Gamma_{1}\right)$ is spanned by

$$
F_{1}=z_{1}^{1 / 5}\left({ }_{2} F_{1}\left(\frac{1}{20}, \frac{1}{4} ; \frac{4}{5} ; z_{1}\right)+C_{1} z_{1}^{1 / 5}{ }_{2} F_{1}\left(\frac{1}{4}, \frac{9}{20} ; \frac{6}{5} ; z_{1}\right)\right)^{8}
$$

for some constant $C_{1}$, and the function

$$
F_{2}=z_{2}^{3 / 5}\left(1-z_{2}\right)^{1 / 5}\left({ }_{2} F_{1}\left(\frac{3}{10}, \frac{2}{5} ; \frac{9}{10} ; z_{2}\right)+C_{2} z_{2}^{1 / 10}{ }_{2} F_{1}\left(\frac{2}{5} ; \frac{1}{2} ; \frac{11}{10} ; z_{2}\right)\right)^{8}
$$

is contained in $S_{8}\left(\Gamma_{2}\right)$ for some constant $C_{2}$. To get a basis for $S_{8}\left(\Gamma_{3}\right)$, we need to work out the Schwarzian differential equation associated to $z_{3}$. It is actually easy in this case.

By Proposition 2, the function $z_{3}^{\prime}(\tau)$, as a function of $z_{3}$, satisfies

$$
\frac{d^{2}}{d z_{3}^{2}} f+Q\left(z_{3}\right) f=0
$$

where

$$
Q\left(z_{3}\right)=\frac{6}{25}\left(\frac{1}{z_{3}^{2}}+\frac{1}{\left(1-z_{3}\right)^{2}}+\frac{1}{\left(1+z_{3}\right)^{2}}\right)+\frac{B_{1}}{z_{3}}+\frac{B_{2}}{z_{3}-1}+\frac{B_{3}}{z_{3}+1}
$$

for some complex numbers satisfying

$$
B_{1}+B_{2}+B_{3}=0, \quad B_{2}-B_{3}+\frac{12}{25}=0 .
$$


To determine the values of $B_{j}$, we use the automorphism of $X_{3}$ coming from the normal subgroup relation $\Gamma_{3} \triangleleft \Gamma_{1}$. Let $\gamma$ be an element of $\Gamma_{2}$ not in $\Gamma_{3}$. We know that

$$
z_{3}(\gamma \tau)=-z_{3}(\tau)
$$

Now by Proposition [5] we have

$$
\begin{aligned}
D\left(-z_{3}(\tau), \tau\right) & =D\left(z_{3}(\gamma \tau), \tau\right)=D\left(z_{3}(\gamma \tau), \gamma \tau\right)+D(\gamma \tau, \tau) /(d \gamma \tau / d \tau)^{2}=Q\left(z_{3}(\gamma \tau)\right) \\
& =\frac{6}{25}\left(\frac{1}{z_{3}^{2}}+\frac{1}{\left(1-z_{3}\right)^{2}}+\frac{1}{\left(1+z_{3}\right)^{2}}\right)+\frac{B_{1}}{-z_{3}}+\frac{B_{2}}{-z_{3}-1}+\frac{B_{3}}{-z_{3}+1}
\end{aligned}
$$

On the other hand, we also have, by the same proposition,

$$
D\left(-z_{3}, \tau\right)=D\left(-z_{3}, z_{3}\right)+D\left(z_{3}, \tau\right) /(-1)^{2}=Q\left(z_{3}\right) .
$$

Comparing (19), (21), and (22), we find $B_{1}=0$ and $B_{2}=-B_{3}$. Together with (20), this gives us

$$
B_{1}=0, \quad B_{2}=-\frac{6}{25}, \quad B_{3}=\frac{6}{25}
$$

and

$$
Q\left(z_{3}\right)=\frac{6}{25}\left(\frac{1}{z_{3}^{2}}+\frac{1}{\left(1-z_{3}\right)^{2}}+\frac{1}{\left(1+z_{3}\right)^{2}}+\frac{1}{1-z_{3}}+\frac{1}{1+z_{3}}\right) .
$$

Now a basis for the solution space of the Schwarzian differential equation $d^{2} f / d z_{3}^{2}+$ $Q\left(z_{3}\right) f=0$ is given by

$$
\begin{aligned}
& f_{1}=z_{3}^{2 / 5}\left(1-\frac{4}{15} z_{3}^{2}-\frac{52}{475} z_{3}^{4}-\frac{13436}{206625} z_{3}^{6}-\frac{46348}{1033125} z_{3}^{8}-\frac{2024924}{60265625} z_{3}^{10}-\cdots\right), \\
& f_{2}=z_{3}^{3 / 5}\left(1-\frac{12}{55} z_{3}^{2}-\frac{28}{275} z_{3}^{4}-\frac{2708}{42625} z_{3}^{6}-\frac{393636}{8738125} z_{3}^{8}-\frac{7503908}{218453125} z_{3}^{10}-\cdots\right) .
\end{aligned}
$$

By Proposition 2,

$$
g, z_{3} g, z_{3}^{2} g, z_{3}^{3} g, z_{3}^{4} g, \quad g=\frac{\left(f_{1}+C_{3} f_{2}\right)^{8}}{z_{3}^{3}\left(1-z_{3}\right)^{3}\left(1+z_{3}\right)^{3}},
$$

form a basis for $S_{8}\left(\Gamma_{3}\right)$ for some constant $C_{3}$. That is, after substituting (15) and (14) into (17) and (18), respectively, we have $F_{1}=h_{1}\left(z_{3}\right) g$ and $F_{2}=h_{2}\left(z_{3}\right) g$ for some polynomials $h_{1}(x)$ and $h_{2}(x)$ of degree $\leq 4$. Indeed, by comparing the coefficients, we find

$$
F_{1}=2^{6 / 5}\left(1-z_{3}-z_{3}^{2}\right)\left(1+4 z_{3}-z_{3}^{2}\right) g, \quad F_{2}=z_{3} g .
$$

(The computation becomes easier if we take the 8th roots of the functions first.) Simplifying the relation $z_{3} F_{1}=2^{6 / 5}\left(1-z_{3}-z_{3}^{2}\right)\left(1+4 z_{3}-z_{3}^{2}\right) F_{2}$, we get the two identities in the theorem. This completes the proof. 


\section{Algebraic transformations associated to other Classes}

5.1. Classes II, V, and XII. The subgroup diagrams of Classes II, V, and XII are all of the form

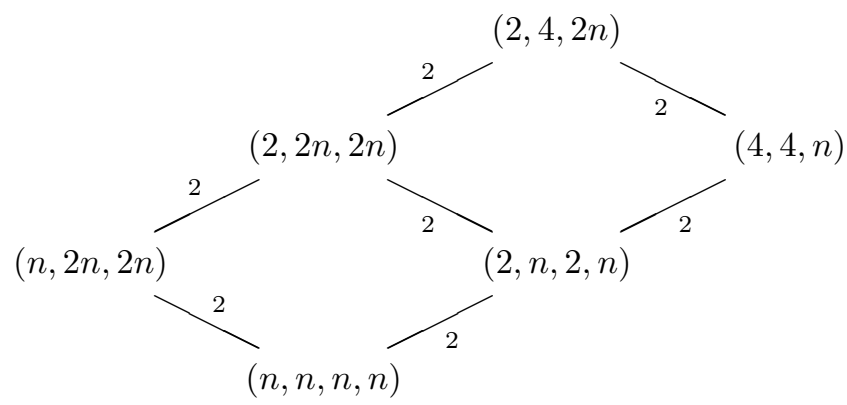

The subgroup relation $(2,2 n, 2 n) \cap(4,4, n)=(2, n, 2, n)$ is a special case of

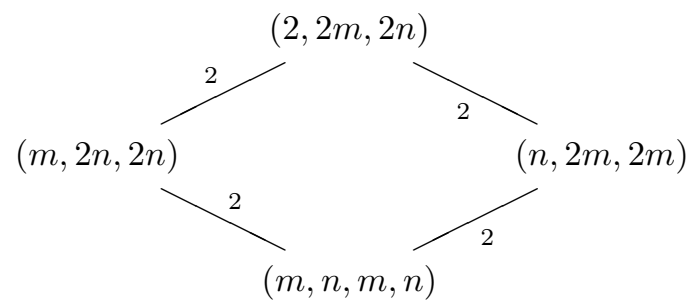

which arises from the Coxeter decompositions of a quadrilateral polygon that is symmetric with respect to both the diagonals as shown below:

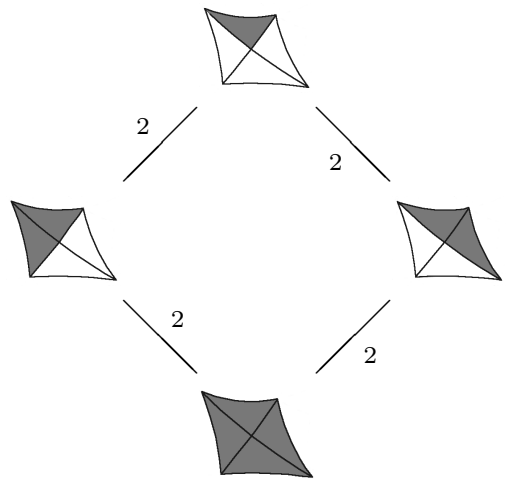

Associated to this family of subgroup relations is the following identity.

Theorem 3. For real numbers $a$ and $b$ such that neither $b+3 / 4$ nor $2 b+1 / 2$ is $a$ nonpositive integer, we have

$$
(1+z)_{2}^{2 a+2 b} F_{1}\left(a+b, a+\frac{1}{4} ; b+\frac{3}{4} ; z^{2}\right)={ }_{2} F_{1}\left(a+b, b+\frac{1}{4} ; 2 b+\frac{1}{2} ; \frac{4 z}{(1+z)^{2}}\right)
$$

in a neighborhood of $z=0$. 
This identity can be easily proved using Kummer's quadratic transformation. Alternatively, one can verify that both sides are solutions of the differential equation $2 z(1-z)(1+z)^{2} F^{\prime \prime}-(1+z)\left((3-4 b) z^{2}+8(a+b) z-4 b-1\right) F^{\prime}-(a+b)(1+4 b)(1-z) F=0$ and that the local behaviors at $z=0$ agree. We omit the details.

5.2. Classes IV, VIII, XI, XIII, XV, and XVII. The subgroup diagrams of Classes IV, VIII, XI, XIII, XV, and XVIII are either of the form

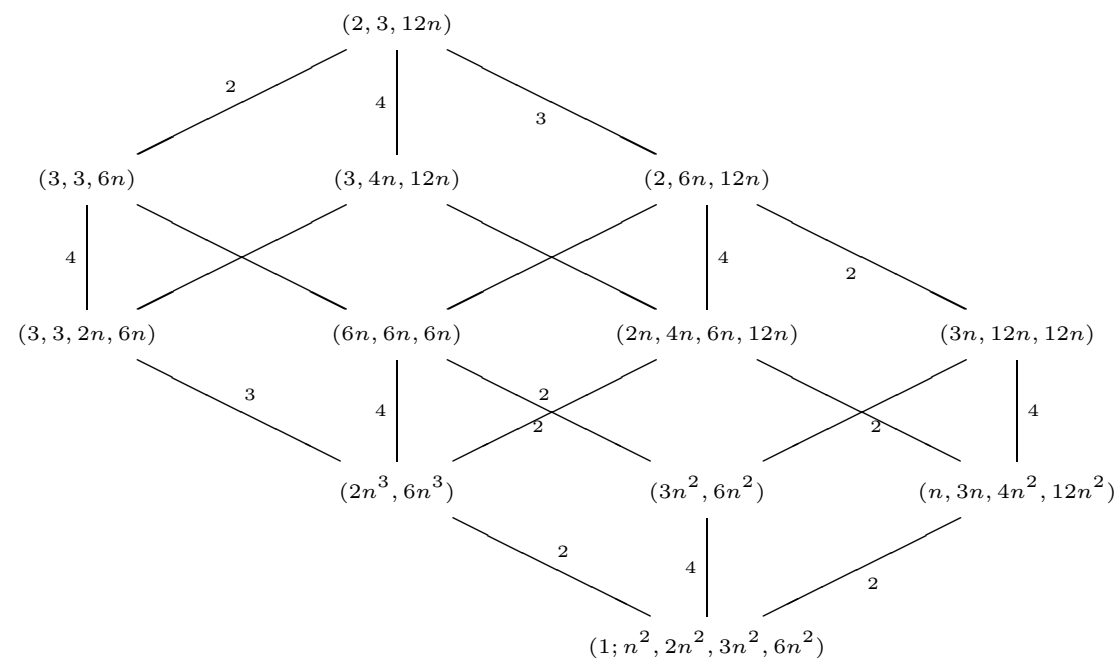

or a subdiagram of it with Class XI having one extra node. There are two families of essentially new identities associated to these classes. One corresponds to the pair of $(3,3,6 n)$ and $(3,4 n, 12 n)$. (Theorem 4 below.) One corresponds to the pair of $(3,4 n, 12 n)$ and $(2,6 n, 12 n)$. (Theorem 5 below.)

Theorem 4. For a real number a such that neither $3 a+1$ nor $2 a+1$ is a nonpositive integer, we have

$$
\begin{gathered}
(1+z)^{a+1 / 6}(1-z / 3)^{3 a+1 / 2}{ }_{2} F_{1}\left(2 a+\frac{1}{3}, a+\frac{1}{3} ; 3 a+1 ; z^{2}\right) \\
={ }_{2} F_{1}\left(a+\frac{1}{6}, a+\frac{1}{2} ; 2 a+1 ; \frac{16 z^{3}}{(1+z)(3-z)^{3}}\right)
\end{gathered}
$$

in a neighborhood of $z=0$.

Theorem 5. For a real number a such that neither $6 a+1$ nor $4 a+1$ is a nonpositive integer, we have

$$
\begin{aligned}
& (1-z)^{9 a+3 / 4}{ }_{2} F_{1}\left(4 a+\frac{1}{3}, 2 a+\frac{1}{3} ; 6 a+1 ;-\frac{27 z^{2}(1-z)}{1-9 z}\right) \\
& =(1-9 z)^{a+1 / 12}{ }_{2} F_{1}\left(3 a+\frac{1}{4}, a+\frac{1}{4} ; 4 a+1 ;-\frac{64 z^{3}}{(1-z)^{3}(1-9 z)}\right)
\end{aligned}
$$

in a neighborhood of $z=0$.

In principle, these two identities can be deduced from Kummer's and Goursat's transformations, once the related Belyi functions are determined. Here we briefly 
indicate how one can prove the theorems in the cases where the parameters correspond to discrete Fuchsian groups using the theory of automorphic forms.

Proof of Theorem 4 in the cases of Shimura curves. For the pair of $(3,3,6 n)$ and $(3,4 n, 12 n)$, the subgroup relations admit Coxeter decompositions, as shown in the figures
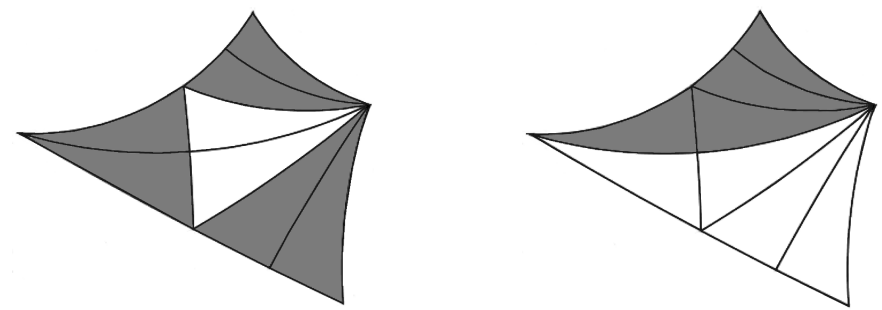

Here the parameter $n$ in the figures is 1 and the smaller triangles are $(2,3,12)$ triangles. Let $\Gamma_{1}=(3,3,6 n), \Gamma_{2}=(3,4 n, 12 n), \Gamma_{3}=\Gamma_{1} \cap \Gamma_{2}$, and let $X_{i}, i=$ $1, \ldots, 3$ be the associated Shimura curves. Denote by $P_{3}, P_{3}^{\prime}$, and $P_{6 n}$ the elliptic points of orders 3,3 , and $6 n$ on $X_{1}$, by $Q_{3}, Q_{4 n}$, and $Q_{12 n}$ the elliptic points of orders $3,4 n$, and $12 n$ on $X_{2}$, and by $R_{3}, R_{3}^{\prime}, R_{2 n}$, and $R_{6 n}$ the elliptic points of order $3,3,2 n$, and $6 n$ on $X_{3}$. The points are labelled in a way such that the ramification data are given by
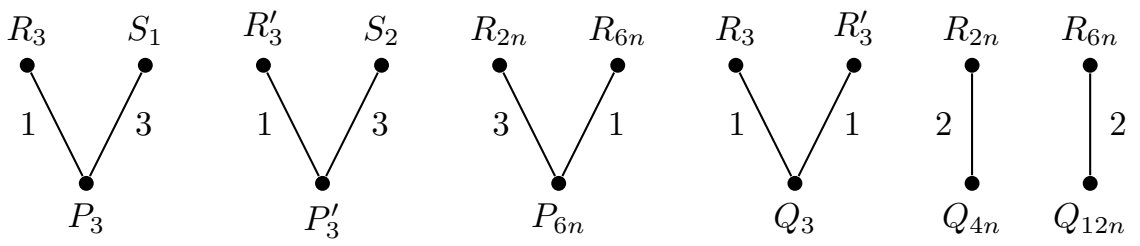

Choose Hauptmoduls $z_{j}$ on $X_{j}, j=1,2,3$, by requiring $z_{1}\left(P_{6 n}\right)=0, z_{1}\left(P_{3}\right)=1, z_{1}\left(P_{3}^{\prime}\right)=\infty, \quad z_{2}\left(Q_{4 n}\right)=0, z_{2}\left(Q_{3}\right)=1, z_{2}\left(Q_{12 n}\right)=\infty$ and

$$
z_{3}\left(R_{2 n}\right)=0, z_{3}\left(R_{3}\right)=1, z_{3}\left(R_{6 n}\right)=\infty .
$$

It is easy to see from the ramification information that

$$
z_{2}=z_{3}^{2}
$$

which implies that $z_{3}\left(R_{3}^{\prime}\right)=-1$. For $z_{1}$, we have

$$
z_{1}=\frac{A z_{3}^{3}}{\left(1+z_{3}\right)\left(1-a z_{3}\right)^{3}}
$$

for some complex numbers $A$ and $a$, where $1 / a$ is the value of $z_{3}$ at $S_{1}$. These two numbers satisfy

$$
1-\frac{A z_{3}^{3}}{\left(1+z_{3}\right)\left(1-a z_{3}\right)^{3}}=1-z_{1}=\frac{\left(1-z_{3}\right)\left(1-b z_{3}\right)^{3}}{\left(1+z_{3}\right)\left(1-a z_{3}\right)^{3}},
$$

where $1 / b$ is the value of $z_{3}$ at $S_{2}$. Now observe that $\Gamma_{3}$ is a normal subgroup of $\Gamma_{2}$. Thus, an element of $\Gamma_{2}$ not in $\Gamma_{3}$ induces an automorphism on $X_{3}$. In terms of the Hauptmodul $z_{3}$, it is easy to see that this automorphism sends $z_{3}$ to $-z_{3}$. 
Since this automorphism maps $S_{1}$ to $S_{2}$, we find $b=-a$. Then comparing the two sides of (24), we get $A=16 / 27, a=1 / 3$, and

$$
z_{1}=\frac{16 z_{3}^{3}}{\left(1+z_{3}\right)\left(3-z_{3}\right)^{3}}
$$

Now by Proposition 1 , we have

$$
\operatorname{dim} S_{6}\left(\Gamma_{1}\right)=\operatorname{dim} S_{6}\left(\Gamma_{2}\right)=1, \quad \operatorname{dim} S_{6}\left(\Gamma_{3}\right)= \begin{cases}2, & \text { if } n=1, \\ 3, & \text { if } n \geq 2 .\end{cases}
$$

From now on, we assume that $n \geq 2$.

By Proposition 4 the one-dimensional spaces $S_{6}\left(\Gamma_{1}\right)$ and $S_{6}\left(\Gamma_{2}\right)$ are spanned by

$$
\begin{aligned}
F_{1}=z_{1}^{1-1 / 2 n} & \left({ }_{2} F_{1}\left(\frac{1}{6}-\frac{1}{12 n}, \frac{1}{2}-\frac{1}{12 n} ; 1-\frac{1}{6 n} ; z_{1}\right)\right. \\
& \left.+C_{1} z_{1}^{1 / 6 n}{ }_{2} F_{1}\left(\frac{1}{6}+\frac{1}{12 n}, \frac{1}{2}+\frac{1}{12 n} ; 1+\frac{1}{6 n} ; z_{1}\right)\right)^{6}
\end{aligned}
$$

and

$$
\begin{aligned}
F_{2}=z_{2}^{1-3 / 4 n} & \left({ }_{2} F_{1}\left(\frac{1}{3}-\frac{1}{6 n}, \frac{1}{3}-\frac{1}{12 n} ; 1-\frac{1}{4 n} ; z_{2}\right)\right. \\
& \left.+C_{2} z_{2}^{1 / 4 n}{ }_{2} F_{1}\left(\frac{1}{3}+\frac{1}{12 n}, \frac{1}{3}+\frac{1}{6 n} ; 1+\frac{1}{4 n} ; z_{2}\right)\right)^{6},
\end{aligned}
$$

respectively, for some constants $C_{1}$ and $C_{2}$. Also, if we let $f_{1}=z_{3}^{1 / 2-1 / 4 n}(1+$ $\left.c_{1} z_{3}+\cdots\right)$ and $f_{2}=z_{3}^{1 / 2+1 / 4 n}\left(1+d_{1} z_{3}+\cdots\right)$ be a basis of the solution space of the Schwarzian differential equation $d^{2} f / d z_{3}^{2}+Q\left(z_{3}\right) f=0$ associated to $z_{3}$, then by Proposition 2. $S_{6}\left(\Gamma_{3}\right)$ is spanned by $g, z_{3} g$, and $z_{3}^{2} g$, where

$$
g=\frac{\left(f_{1}+C_{3} f_{2}\right)^{6}}{z_{3}^{2}\left(1-z_{3}\right)^{2}\left(1+z_{3}\right)^{2}}
$$

for some constant $C_{3}$. Now we substitute (25) and (23) into (26) and (23), respectively. We find

$$
F_{1}=a_{1} z_{3}^{3-3 / 2 n}+\cdots, \quad F_{2}=z_{3}^{2-3 / 2 n}+\cdots,
$$

where $a_{1}=(16 / 27)^{1-1 / 2 n}$, and thus

$$
F_{1}=a_{1} z_{3}^{2} g, \quad F_{2}=\left(z_{3}+a_{2} z_{3}^{2}\right) g
$$

for some constant $a_{2}$. That is, $a_{1} z_{3} F_{2} / F_{1}=1+a_{2} z_{3}$. We then take the 6 th roots of the two sides and compare the coefficients of $z^{2 / 3-1 / 4 n}$; we find that $a_{2}$ is actually 0 . After simplifying, we arrive at

$$
\begin{gathered}
(1+z)^{1 / 6-1 / 12 n}(1-z / 3)^{1 / 2-1 / 4 n}{ }_{2} F_{1}\left(\frac{1}{3}-\frac{1}{6 n}, \frac{1}{3}-\frac{1}{12 n} ; 1-\frac{1}{4 n} ; z^{2}\right) \\
={ }_{2} F_{1}\left(\frac{1}{6}-\frac{1}{12 n}, \frac{1}{2}-\frac{1}{12 n} ; 1-\frac{1}{6 n} ; \frac{16 z^{3}}{(1+z)(3-z)^{3}}\right) .
\end{gathered}
$$

This proves Theorem 4 in the case where the parameters correspond to arithmetic triangle groups. 
Proof of Theorem 5 in the cases of Shimura curves. The subgroups $(3,4 n, 12 n)$, $(2,6 n, 12 n)$ and their intersection admit Coxeter decompositions as the figures below show:
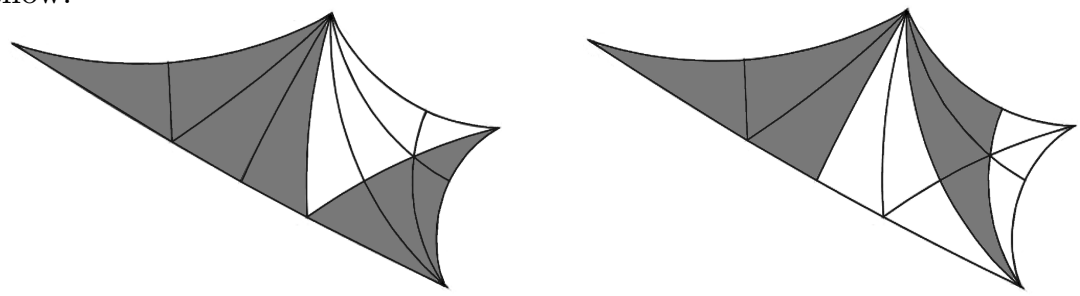

Here the parameter $n$ in the figures is 1 and the small triangles are $(2,3,12)$ triangles.

Denote the groups $(3,4 n, 12 n),(2,6 n, 12 n)$, and $(2 n, 4 n, 6 n, 12 n)$ by $\Gamma_{1}, \Gamma_{2}$, and $\Gamma_{3}$, respectively. Label the elliptic points of $(3,4 n, 12 n)$ by $P_{3}, P_{4 n}$, and $P_{12 n}$, those of $(2,6 n, 12 n)$ by $Q_{2}, Q_{6 n}$, and $Q_{12 n}$, and those of $(2 n, 4 n, 6 n, 12 n)$ by $R_{2 n}, R_{4 n}$, $R_{6 n}$, and $R_{12 n}$. The ramifications are shown as follows:
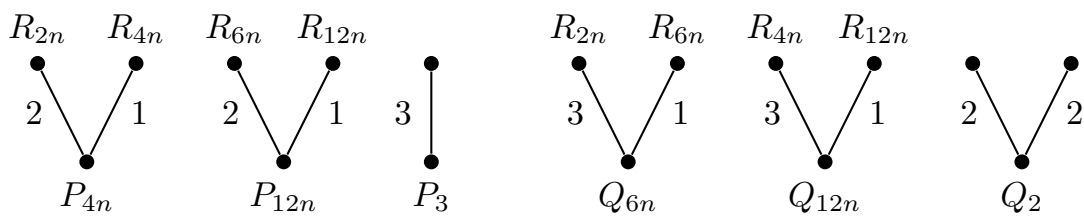

Choose Hauptmoduls $z_{j}$ for $\Gamma_{j}, j=1, \ldots, 3$, by requiring that

$$
\begin{aligned}
& z_{1}\left(P_{4 n}\right)=0, \quad z_{1}\left(P_{3}\right)=1, \quad z_{1}\left(P_{12 n}\right)=\infty, \\
& z_{2}\left(Q_{6 n}\right)=0, \quad z_{2}\left(Q_{2}\right)=1, \quad z_{2}\left(Q_{12 n}\right)=\infty, \\
& z_{3}\left(R_{2 n}\right)=0, \quad z_{3}\left(R_{4 n}\right)=1, \quad z_{3}\left(R_{6 n}\right)=\infty .
\end{aligned}
$$

It is easy to work out the relation between $z_{1}$ and $z_{3}$ and that between $z_{2}$ and $z_{3}$. They are

$$
z_{1}=\frac{27 z_{3}^{2}\left(1-z_{3}\right)}{1-9 z_{3}}, \quad z_{2}=-\frac{64 z_{3}^{3}}{\left(1-z_{3}\right)^{3}\left(1-9 z_{3}\right)} .
$$

Here $1 / 9$ is the value of $z_{3}$ at $R_{12 n}$. We then follow the same arguments as before to obtain the claimed identities. We omit the details.

\section{Appendix A. List of ARIThMetic TRIANGLE GROUPS}

In this section, we determine the signatures of the intersections of commensurable triangle groups.

According to [7, 8], there are totally 85 arithmetic triangle groups, falling in 19 different commensurability classes. Here we give the subgroup diagrams. Note that since most groups here have genus 0 , we omit the genus information from the signature, unless the group has a positive genus. Also, to save space, the notation $\left(g ; e_{1}^{n_{1}}, \ldots, e_{r}^{n_{r}}\right)$ means that the Shimura curve has $n_{i}$ elliptic points of order $e_{i}$. Furthermore, for convenience, we will often call the groups by their signatures, even though this raises some ambiguity.

Remark 11. There is some ambiguity when we say "the intersections of commensurable triangle groups" because there may be more than one order whose norm-one groups have the same signature and the intersections of these groups with another 
group may have different signatures. For example, in the case $B=M(2, \mathbb{Q})$, the subgroups $\Gamma_{0}(2)$ and $\Gamma^{0}(2)$ of $\operatorname{SL}(2, \mathbb{Z})$ have the same signature $(0 ; 2, \infty, \infty)$, and the group $\Gamma_{0}(4)$ has signature $(0 ; \infty, \infty, \infty)$. The intersection of $\Gamma_{0}(2)$ and $\Gamma_{0}(4)$ is just $\Gamma_{0}(4)$, but the intersection of $\Gamma^{0}(2)$ and $\Gamma_{0}(4)$ has signature $(0 ; \infty, \infty, \infty, \infty)$. Thus, the subgroup diagrams described here should be read as "there are arithmetic groups whose subgroup relations are given by the subgroup diagrams".

Since it is not easy to explicitly describe the orders associated to arithmetic triangle groups, here we use group theory and properties of discrete subgroups of $\mathrm{SL}(2, \mathbb{R})$ to determine the signatures. We will work out the case of Class IV in 8 and omit the proof of the others.

According to [8], Class IV of arithmetic triangle groups has the following subgroup diagram:

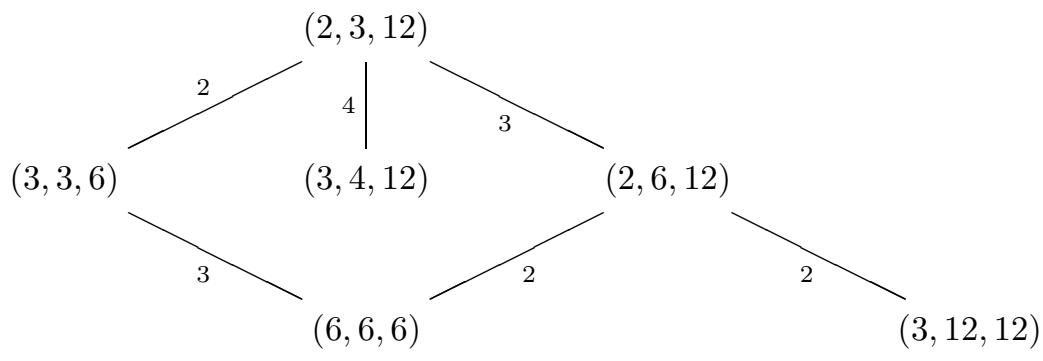

Here the numbers next to the lines are the indices. Set

$$
\begin{array}{lll}
\Gamma_{1}=(2,3,12), & \Gamma_{2}=(3,3,6), & \Gamma_{3}=(3,4,12), \\
\Gamma_{4}=(2,6,12), & \Gamma_{5}=(6,6,6), & \Gamma_{6}=(3,12,12),
\end{array}
$$

and let $X_{i}, i=1, \ldots, 6$, denote the respective Shimura curves. To determine $\Gamma_{2} \cap \Gamma_{3}$, we observe that $\Gamma_{2}$ is a normal subgroup of $\Gamma_{1}$ of index 2 and $\Gamma_{1}=\Gamma_{2} \Gamma_{3}$. Thus, $\Gamma_{2} \cap \Gamma_{3}$ is a normal subgroup of $\Gamma_{3}$ of index 2. Now the elliptic point of order 3 on $X_{3}$ must split into two points in $X\left(\Gamma_{2} \cap \Gamma_{3}\right)$ because $2 \nmid 3$. Then from the Riemann-Hurwitz formula, we see that the elliptic points of order 4 and 12 must be ramified. That is, the curve $X\left(\Gamma_{2} \cap \Gamma_{3}\right)$ must have signature $(2,3,3,6)$. In fact, this can also be seen from the following figures:
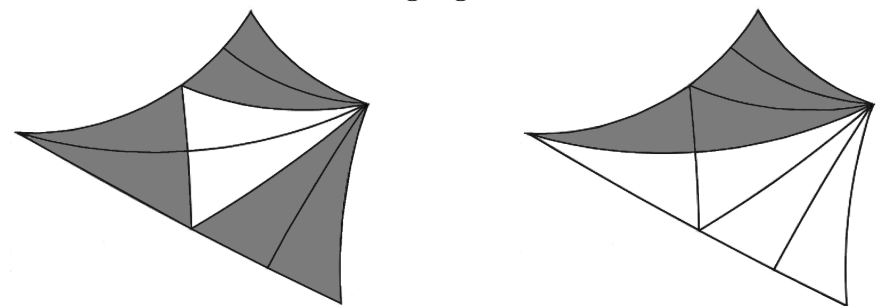

Here the smaller triangles are $(2,3,12)$-triangles. The figures show that the triangle group $(2,3,12)$ contains two subgroups of signatures $(3,3,6)$ and $(3,4,12)$, respectively, whose intersection has signature $(2,3,3,6)$. (In fact, the theoretical argument above shows that for any pair of subgroups of $\Gamma_{1}$ with signatures $(3,3,6)$ and $(3,4,12)$, respectively, the intersection must have signature $(2,3,3,6)$.) 
Likewise, the figures
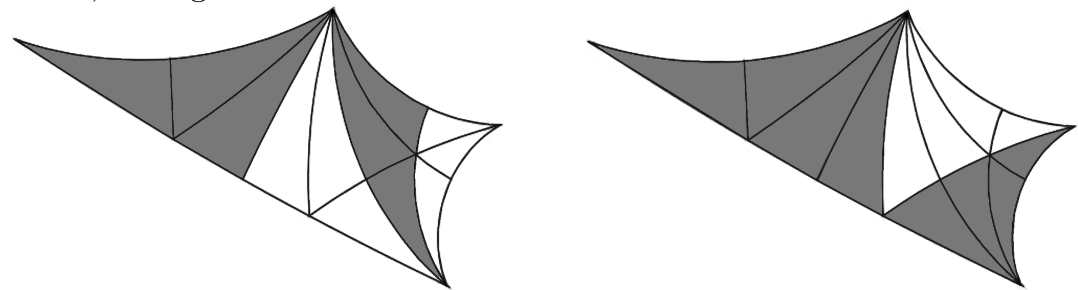

show that there are two subgroups of $\Gamma_{1}$ of signatures $(2,6,12)$ and $(3,4,12)$ such that their intersection has signature $(2,4,6,12)$. We have the following subgroup diagram:

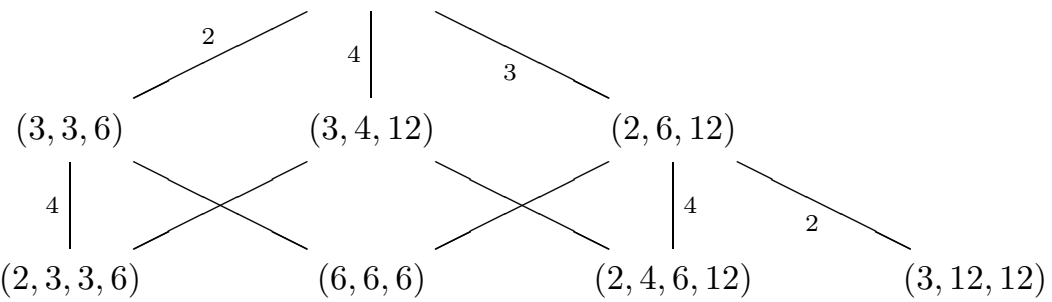

Let $\Gamma_{7}=(2,3,3,6)$ and $\Gamma_{8}=(2,4,6,12)$ and $X_{7}$ and $X_{8}$ be their associated Shimura curves. Again, because $\Gamma_{5}$ is a normal subgroup of $\Gamma_{4}$ of index 2 and $\Gamma_{5} \Gamma_{8}=\Gamma_{4}$, the intersection of $\Gamma_{5}$ and $\Gamma_{8}$ is a subgroup of index 2 of $\Gamma_{8}$. Now the group $(2,4,6,12)$ has many subgroups of index 2. (The structure of the quotient group of $(2,4,6,12)$ over its commutator subgroup is $C_{2} \times C_{4} \times C_{6}$.) To determine which of them is contained in the group $(6,6,6)$, we use the following properties.

(1) If $p$ is an elliptic point of order $e$ on $X_{8}$, then its preimage in the covering $X\left(\Gamma_{5} \cap \Gamma_{8}\right) \rightarrow X_{8}$ consists of either a single elliptic point of order $e / 2$ or two elliptic points of order $e$.

(2) The total branch number of any finite covering of a compact Riemann surface is always even.

(3) The volume of $X\left(\Gamma_{5} \cap \Gamma_{8}\right)$ is twice of that of $X_{8}$. Thus, if $\left(g ; e_{1}, \ldots, e_{r}\right)$ is the signature of $X\left(\Gamma_{5} \cap \Gamma_{8}\right)$, then we must have

$$
2 g-2+\sum_{i=1}^{r}\left(1-\frac{1}{e_{j}}\right)=2\left(2-\frac{1}{2}-\frac{1}{4}-\frac{1}{6}-\frac{1}{12}\right)=2 .
$$

From this information, we find that possible signatures of a subgroup of index 2 of $(2,4,6,12)$ are

$$
\begin{aligned}
& (1 ; 2,3,6),\left(0 ; 2,6^{2}, 12^{2}\right),\left(0 ; 3,4^{2}, 12^{2}\right),\left(0 ; 4^{2}, 6^{3}\right), \\
& \left(0 ; 2^{3}, 3,12^{2}\right),\left(0 ; 2^{3}, 6^{3}\right),\left(0 ; 2^{2}, 3,4^{2}, 6\right) .
\end{aligned}
$$

Likewise, an elliptic point of order 6 on $X_{5}$ can

(1) split into 4 elliptic points of order 6 , or

(2) split into 2 elliptic points of order 3 , or 
(3) split into 1 elliptic point of order 3 and 2 elliptic point of order 6 , or

(4) split into 1 elliptic point of order 2 and 1 elliptic point of order 6

in the covering $X\left(\Gamma_{5} \cap \Gamma_{8}\right) \rightarrow X_{5}$ of degree 4. Also, the total branch number of $X\left(\Gamma_{5} \cap \Gamma_{8}\right) \rightarrow X_{5}$ must be a positive even integer and the volume of $X\left(\Gamma \cap \Gamma_{8}\right)$ is 2. We find the possible signatures of a subgroup of index 4 of $\Gamma_{5}$ are

$$
\left(0 ; 2^{3}, 6^{3}\right),\left(0 ; 2^{2}, 3^{2}, 6^{2}\right),\left(0 ; 2,3^{4}, 6\right),\left(0 ; 3^{6}\right) .
$$

From (29) and (30), we conclude that the signature of $\Gamma_{5} \cap \Gamma_{8}$ must be $\left(0 ; 2^{3}, 6^{3}\right)$. This can also be seen from the following figures:
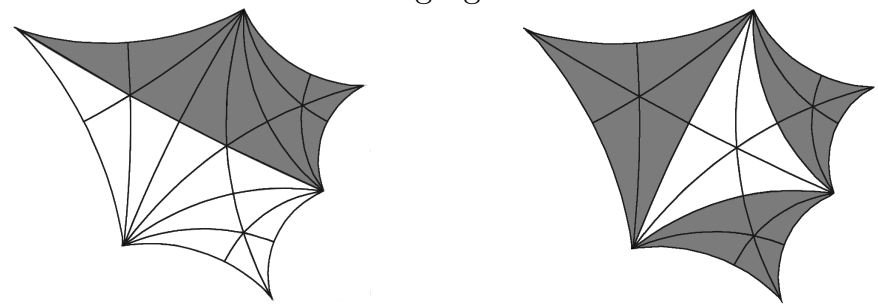

By the same argument, we can also show that the intersection of $\Gamma_{6}$ and $\Gamma_{8}$ must have signature $\left(0 ; 3,4^{2}, 12^{2}\right)$, and the intersection of $\Gamma_{5}$ and $\Gamma_{6}$ has signature $(0 ; 3,3,6,6)$. The subgroup diagram becomes

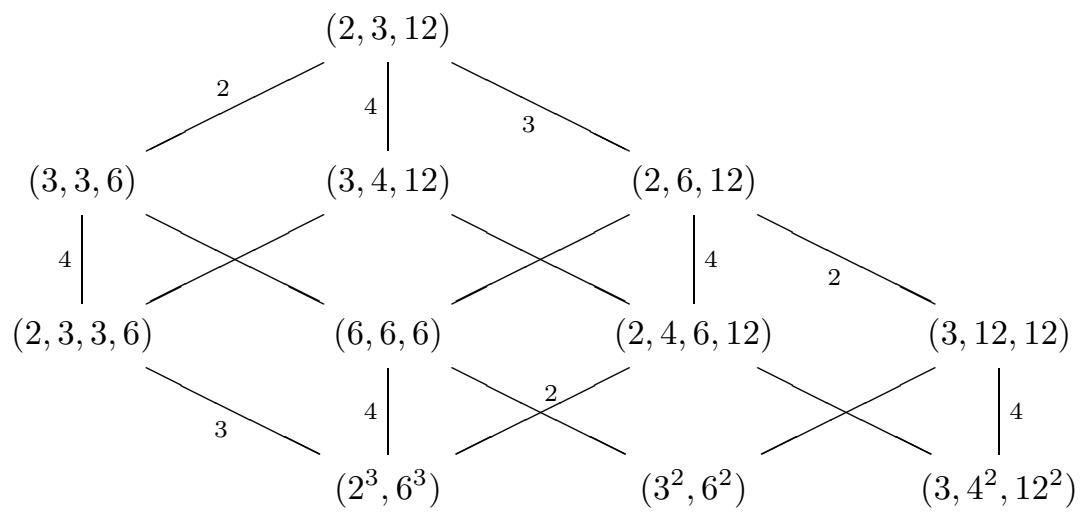

Finally, we can show that the only possible signatures of subgroups of index 2 in $\left(2^{3}, 6^{3}\right)$ are

$$
\left(0 ; 2^{6}, 3^{2}, 6^{2}\right),\left(0 ; 2^{4}, 3,6^{4}\right),\left(0 ; 2^{2}, 6^{6}\right),\left(1 ; 2^{4}, 3^{3}\right),\left(1 ; 2^{2}, 3^{2}, 6^{2}\right),\left(1 ; 3,6^{4}\right),\left(2 ; 3^{3}\right),
$$

while the only possible signatures of subgroups of index 2 in $\left(3,4^{2}, 12^{2}\right)$ are

$$
\left(0 ; 3^{2}, 4^{4}, 6^{2}\right),\left(0 ; 2,3^{2}, 4^{2}, 6,12^{2}\right),\left(0 ; 2^{2}, 3^{2}, 12^{4}\right),\left(1 ; 2^{2}, 3^{2}, 6^{2}\right) .
$$

From these, we see that the common intersection of $\left(2^{3}, 6^{3}\right),\left(3,4^{2}, 12^{2}\right)$, and $\left(3^{2}, 6^{2}\right)$ has signature $\left(1 ; 2^{2}, 3^{2}, 6^{2}\right)$. This completes the proof of the case of Class IV.

Now we give the subgroup diagrams for arithmetic triangle groups. 
5.3. Class II.

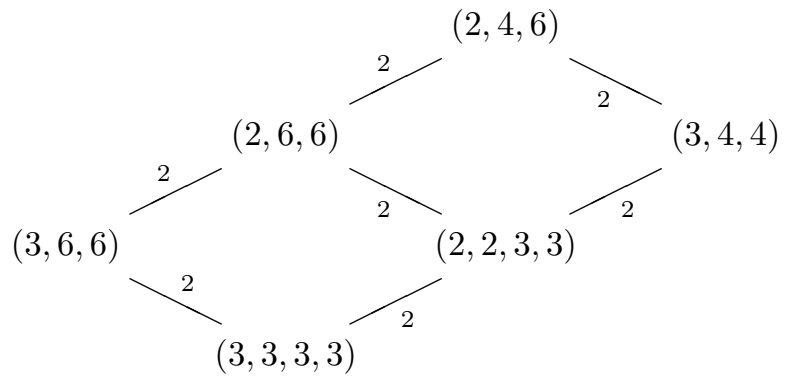

\section{Class III.}

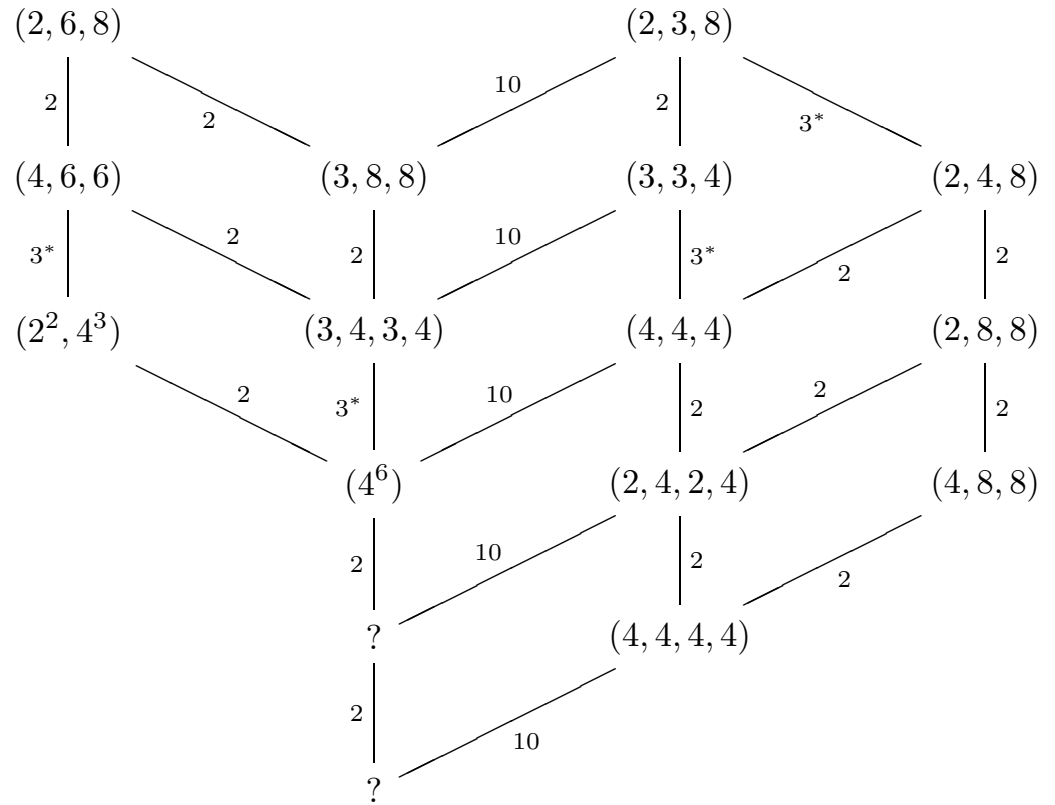




\section{Class IV.}

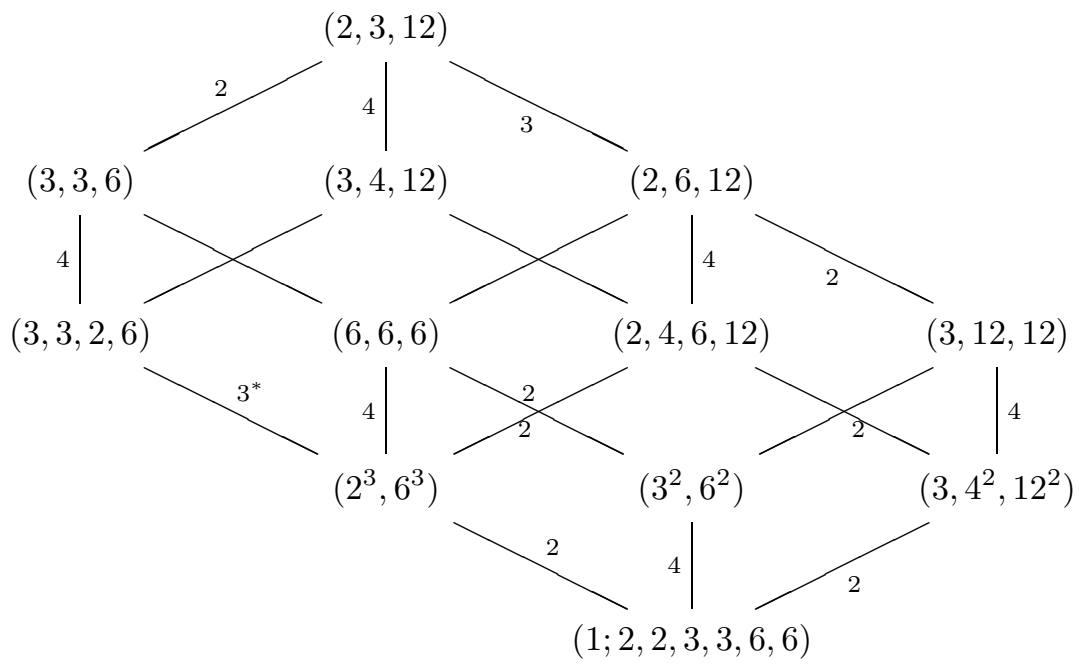

Class V.

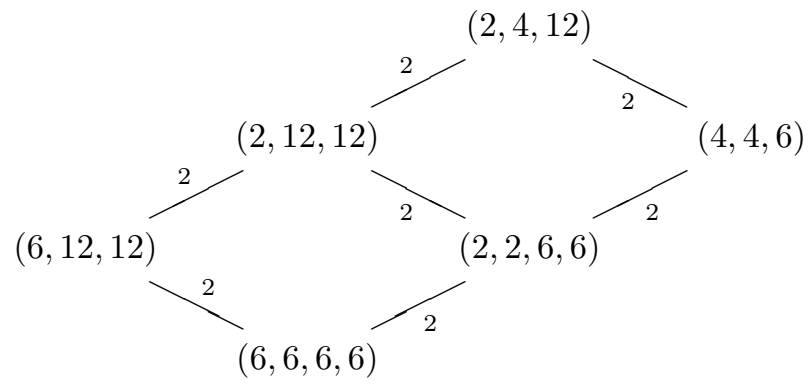

Class VI.

$(2,4,5)$

$(2,4,10)$

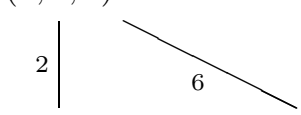

$(2,5,5)$

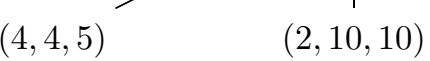

$6^{*}{ }^{*}$

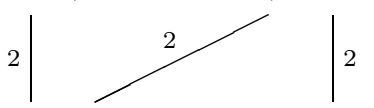

$(2,2,5,5)$

$(5,10,10)$
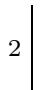

$(5,5,5,5)$

\section{Class VII.}

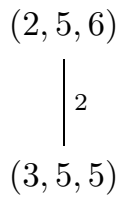




\section{Class VIII.}

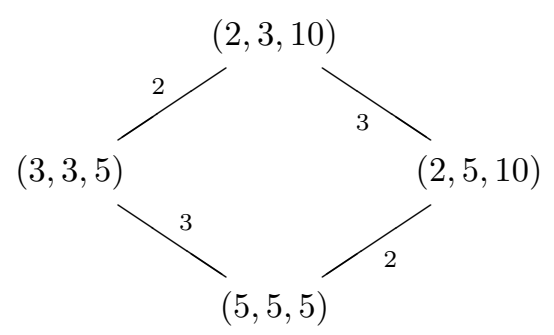

Class IX.

$$
(3,4,6)
$$

\section{Class X.}

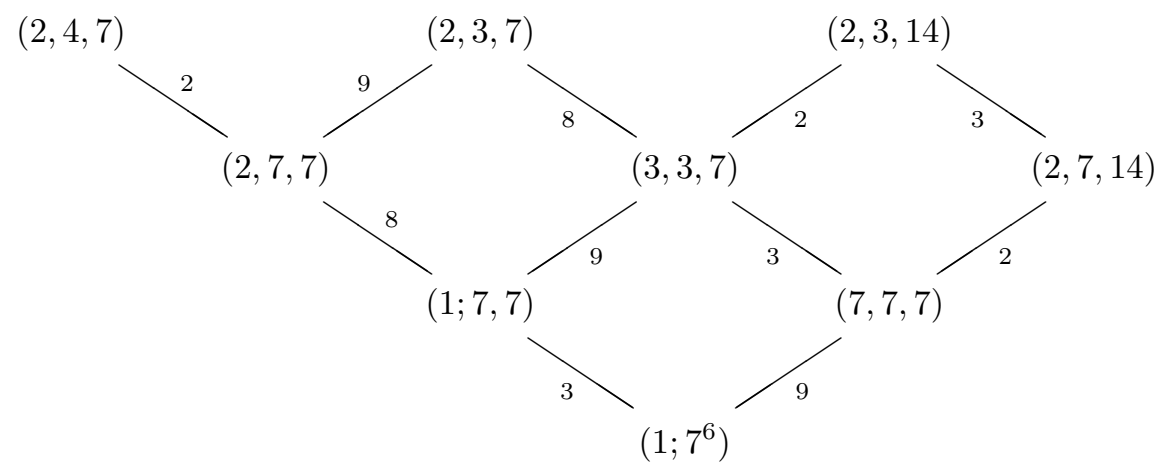

\section{Class XI.}

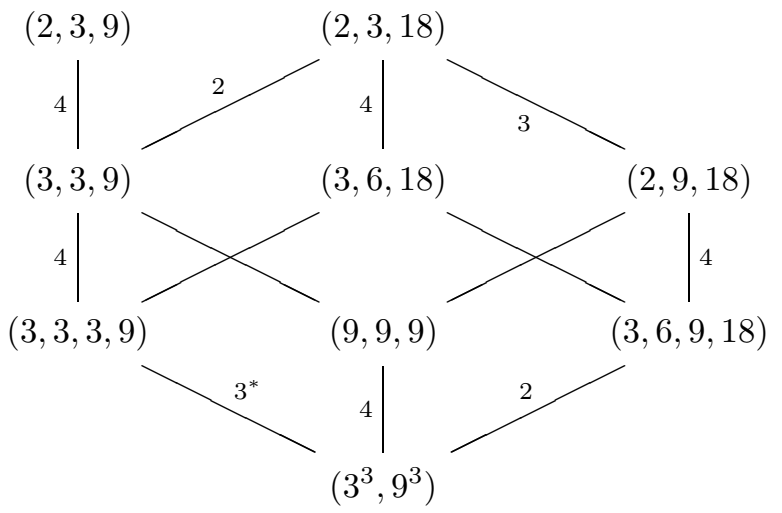




\section{Class XII.}

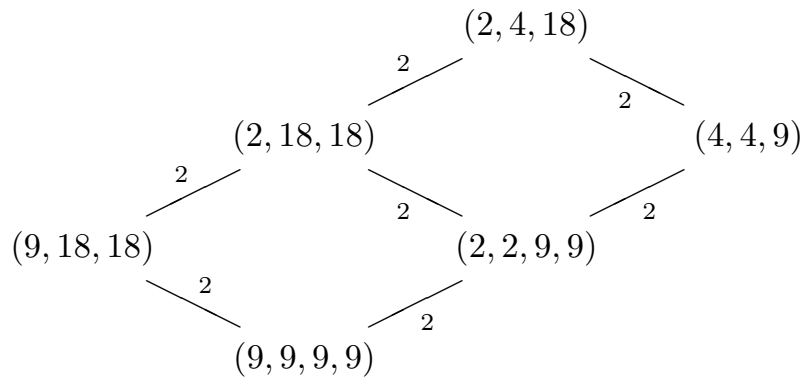

Class XIII.

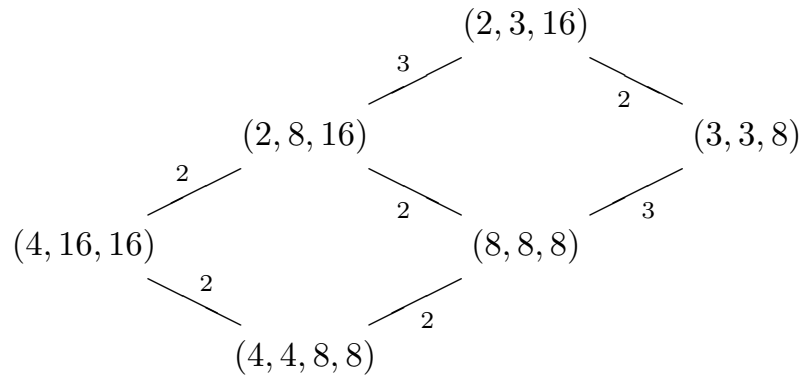

Class XIV.

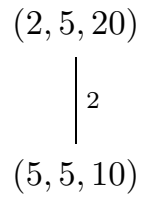

\section{Class XV.}

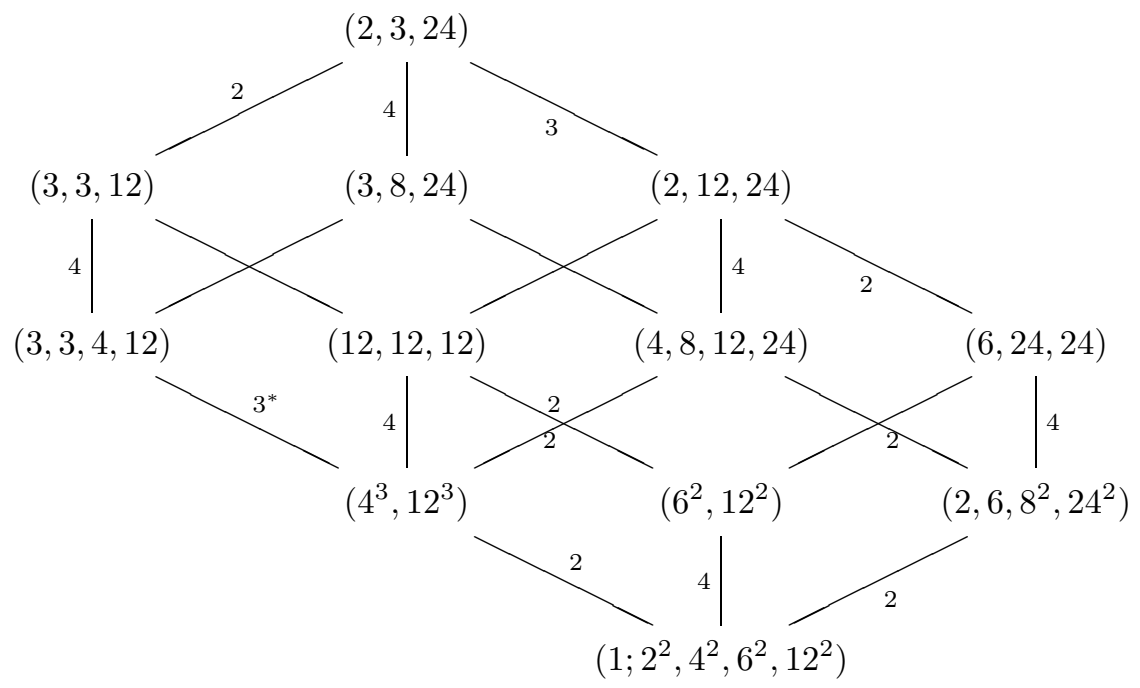


Class XVI.

\section{Class XVII.}

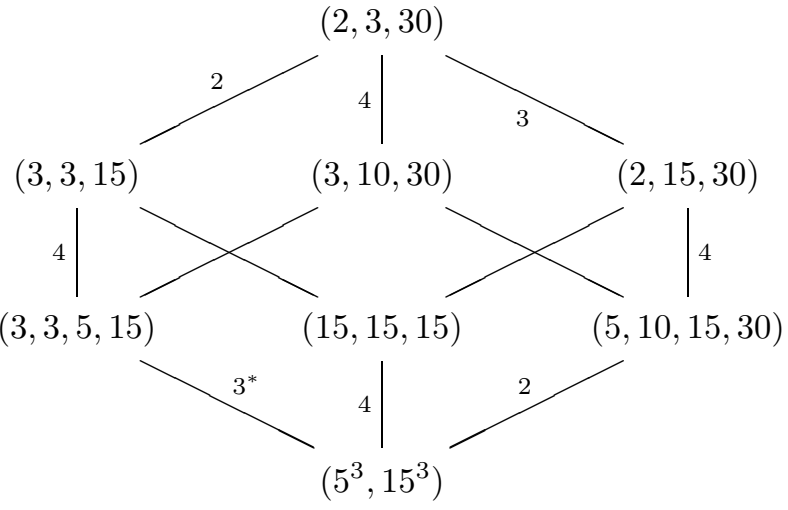

Class XVIII.

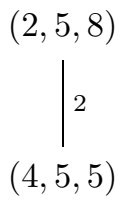

\section{Class XIX.}

\section{REFERENCES}

1. Pilar Bayer and Artur Travesa, Uniformizing functions for certain Shimura curves, in the case $D=6$, Acta Arith. 126 (2007), no. 4, 315-339. MR2289964 (2008d:11055)

2. Noam D. Elkies, Shimura curves for level-3 subgroups of the $(2,3,7)$ triangle group, and some other examples, Algorithmic number theory, Lecture Notes in Comput. Sci., vol. 4076, Springer, Berlin, 2006, pp. 302-316. MR2282932 (2007i:11082)

3. Édouard Goursat, Sur l'équation différentielle linéaire, qui admet pour intégrale la série hypergéométrique, Ann. Sci. École Norm. Sup. 10 (1881), 3-142. MR1508709

4. Svetlana Katok, Fuchsian groups, Chicago Lectures in Mathematics, University of Chicago Press, Chicago, IL, 1992. MR1177168 (93d:20088)

5. Goro Shimura, Construction of class fields and zeta functions of algebraic curves, Ann. of Math. (2) 85 (1967), 58-159. MR.0204426 (34:4268)

6. Introduction to the arithmetic theory of automorphic functions, Publications of the Mathematical Society of Japan, vol. 11, Princeton University Press, Princeton, NJ, 1994. Reprint of the 1971 original, Kano Memorial Lectures, 1. MR.1291394 (95e:11048)

7. Kisao Takeuchi, Arithmetic triangle groups, J. Math. Soc. Japan 29 (1977), no. 1, 91-106. MR0429744(55:2754)

8. Commensurability classes of arithmetic triangle groups, J. Fac. Sci. Univ. Tokyo Sect. IA Math. 24 (1977), no. 1, 201-212. MR0463116 (57:3077)

9. Fang-Ting Tu, Schwarzian differential equations associated to Shimura curves of genus zero, preprint (2011). 
10. Raimundas Vidūnas, Transformations of some Gauss hypergeometric functions, J. Comput. Appl. Math. 178 (2005), no. 1-2, 473-487. MR2127899(2006a:33003)

11. Marie-France Vignéras, Arithmétique des algèbres de quaternions, Lecture Notes in Mathematics, vol. 800, Springer, Berlin, 1980. MR.580949 (82i:12016)

12. Yifan Yang, Schwarzian differential equations and Hecke eigenforms on Shimura curves, Compositio Math. (to appear).

Department of Applied Mathematics, National Chiao Tung University, 1001 Ta Hsueh Road, Hsinchu, Taiwan 30010, Republic of China

E-mail address: ft12.am95g@nctu.edu.tw

Department of Applied Mathematics, National Chiao Tung University, 1001 Ta Hsueh Road, Hsinchu, Taiwan 30010, Republic of China - And - National Center for Theoretical Sciences, Hsinchu 300, Taiwan, Republic of China

E-mail address: yfyang@math.nctu.edu.tw 